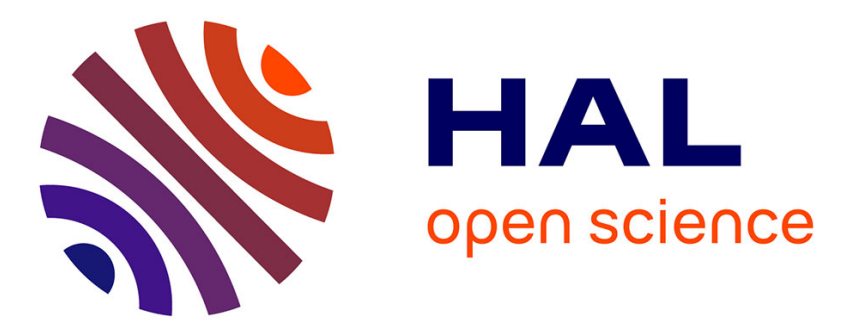

\title{
Impact of group size and social composition on group vocal activity and acoustic network in a social songbird
}

Marie S.A. Fernandez, Clémentine Vignal, Hédi Soula

\section{To cite this version:}

Marie S.A. Fernandez, Clémentine Vignal, Hédi Soula. Impact of group size and social composition on group vocal activity and acoustic network in a social songbird. Animal Behaviour, 2017, 127, pp.163-178. 10.1016/j.anbehav.2017.03.013 . hal-01537565

\section{HAL Id: hal-01537565 \\ https://hal.science/hal-01537565}

Submitted on 27 Jun 2017

HAL is a multi-disciplinary open access archive for the deposit and dissemination of scientific research documents, whether they are published or not. The documents may come from teaching and research institutions in France or abroad, or from public or private research centers.
L'archive ouverte pluridisciplinaire HAL, est destinée au dépôt et à la diffusion de documents scientifiques de niveau recherche, publiés ou non, émanant des établissements d'enseignement et de recherche français ou étrangers, des laboratoires publics ou privés. 
Impact of group size and social composition on group

vocal activity and acoustic network in a social songbird

M.S.A. Fernandez ${ }^{1,2}$, C. Vignal ${ }^{1,}$ H.A. Soula ${ }^{2,3}$

1 Univ Lyon, UJM-Saint-Etienne, CNRS, Neuro-PSI/ENES UMR9197, France

2 EPI BEAGLE INRIA, Villeurbanne, France

3 INSERM U1060 INSA, Villeurbanne, France

Correspondence:

M.S.A. Fernandez

marieferndez@gmail.com

Word Count $=10759$

Keywords = pair-bond, zebra finch, acoustic communication, social network, group collective calling, turn-taking, vocalizations, songbird. 
Abstract

In social species individuals living in the same group may synchronize activities such as movements, foraging or anti-predator vigilance. Synchronization of activities can also be observed between partners especially during breeding and can be crucial for breeding success. Vocalizations are behaviours that can be coordinated between individuals, but simultaneous vocalizations in groups have most of the time been considered as a noise that does not bear any

8 information.

9 Indeed little is known about the structure and function of vocal communications involving a network 10 of individuals. How the individual vocal activity participates in the resulting communal sound, and 11 in return how the group influences individual vocal activity, are questions that remain to be studied.

12 Zebra finches are social monogamous songbirds that form lifelong pair-bonds. In the wild, zebra

13 finches are typically found in small groups, with the pair as the primary social unit, and gather in

14 'social' trees where both females and males produce vocalizations. Here we investigated in the 15 laboratory the influence of group size and composition on general vocal activity and synchrony, as

16 well as the influence of pair-bond and spatial location on finer characteristics of

17 dyads vocal interactions. We used a set-up that locked the birds at fixed spatial positions of our 18 choosing to control the proximity network and allowed us an individual tagging of most of the 19 vocalizations. We used an in-house software suite that automatically detects vocalizations from 20 hours of passive recording.

21 We show that zebra finches groups synchronize their general vocal activity with waves of collective 22 vocalizations, which depend both on the group size and composition. The acoustic network is 23 shaped by pair-bonds at different time scales. Birds preferentially vocalize closely in time 24 (synchrony) or directly after (turn-taking) their partner when it is present and then the nearest 25 neighbour when the partner is not available.

\section{Introduction}


27 In social species, many activities are synchronized between individuals living in the same group.

28 Group members maintain group cohesion during movements and foraging (Agetsuma, 1995;

29 Beauchamp, 1992; Blanc \& Thériez, 1998; Blanc, Thériez, \& Brelurut, 1999; Boyd \& Bandi, 2002;

30 Chivers, 1974; Conradt, 1998; Côte, Schaefer, \& Messier, 1997; Daan \& Slopsema, 1978;

31 Gillingham \& Klein, 1992; Linnane, Brereton, \& Giller, 2001; McMahon \& Evans, 1992;

32 Rasmussen, 1985; Rook \& Huckle, 1995; Ruckstuhl, 1998, 1999; Ruckstuhl \& Neuhaus, 2001;

33 Saino, Fasola, \& Waiyaki, 1995; Schenkeveld \& Ydenberg, 1985; Tayler, 1953; Tremblay \&

34 Cherel, 1999; Wilson, Wilson, \& McQuaid, 1986). Individuals may also coordinate activities such as anti-predator vigilance and feeding (Gerkema \& Verhulst, 1990; Kavanagh, 1978; Rook \& Penning, 1991). Activities can also be synchronized between partners of a pair. Pairs in many long-term monogamous species show an increase in their breeding success over time, and this could be attributed to a better coordination of partners (Black \& Hulme, 1996; Forslund \& Pärt, 1995). Partners can also coordinate foraging activities, and can synchronize their nest visits to feed the chicks (Bebbington \& Hatchwell, 2015; Lee, Kim, \& Hatchwell, 2010; Mariette \& Griffith, 2012, 2015; van Rooij \& Griffith, 2013). When both partners incubate, their hatching success can increase by synchronizing foraging trips (Coulson, 1966; Davis, 1988) or coordinating incubation bouts (Spoon, Millam, \& Owings, 2006). maintenance (Farabaugh, 1982; Hall, 2004) or parental care (Boucaud, Mariette, Villain, \& Vignal, 2015; Elie et al., 2010). Some group vocal productions have also been identified as organized signals bearing messages, like the communal vocalizations of some social mammals that

Vocalizations are behaviours that can be coordinated between individuals. Territorial songbirds vocally compete by answering each other, sometimes matching their song-types and using songoverlap as a signal of escalation (Langemann, Tavares, Peake, \& McGregor, 2000). Some monogamous birds use coordinated vocal duets for territory defense, mate guarding, pair-bond communicate on spacing (Bornean gibbons, Hylobates muelleri, (Mitani, 1984); wolves, Canis 
53 lupus, (Harrington \& Mech, 1979); (Frommolt, 1999)) or group size (female lions, Leo leo,

54 (McComb, Packer, \& Pusey, 1994). But most of the time, group vocal productions have been considered as by-products of individuals' simultaneous but not necessarily coordinated vocalizations. For example, this noisy sound can result from the activity of up to thousands of

57 individuals in choruses of birds (Burt \& Vehrencamp, 2005), insects (Greenfield, 1994), frogs (M.

58 E. Bates, Cropp, Gonchar, \& Knowles, 2010; Jones, Jones, \& Ratman, 2009; Marshall, 2003;

59 Simmons, Bates, \& Knowles, 2009), as well as in fish communities (D'spain \& Berger, 2004;

60 Locascio, 2004; Locascio \& Mann, 2005; Mann, 2003), colonies of nesting birds (Adret-

61 Hausberger, 1982; Mathevon, 1997) or breeding marine mammals (Schusterman, 1978; Southall, 62 Schusterman, \& Kastak, 2003). This sound resulting from a group of individuals vocalizing 63 simultaneously has mainly been viewed as a source of noise pollution constraining the pairwise 64 communications (Aubin \& Jouventin, 1998; Gerhardt \& Klump, 1988). But this group sound with no 65 clear identifiable message might bear information on the structure of the underlying social 66 network (McGregor \& Horn, 2014).

Not much is known about the structures and functions of vocal communications involving a network of individuals. More specifically, little is known about the dynamics of pairs and group vocal exchanges at an individual-level resolution. How the individual vocal activity participates in the resulting communal sound, and in return how the group influences individual vocal activity, are questions that remain to be studied. Yet, we know that the group, as a communication network, is composed of several signalers and receivers sharing the same active signaling space, which implies that it can interfere with pairwise vocal exchanges. Eavesdropping is defined as extracting information from signaling interactions while not being the main recipient, and seems to occur in many species (McGregor \& Dabelsteen, 1996). In birds for example, it has been shown that eavesdroppers can respond to vocal exchanges even if they were not part of it initially (Mennill, Ratcliffe, \& Boag, 2002). Audience effects show that the presence of other conspecifics can 
79 influence a sender's behavior (Doutrelant, McGregor, \& Oliveira, 2001; Evans \& Marler, 1994;

80 Hector, Seyfarth, \& Raleigh, 1989; Matos \& McGregor, 2002; Plath, Blum, Schlupp, \& Tiedemann,

81 2008). The communication behaviour of male zebra finches can be modified by the individuals that

82 are listening, and by the nature of the social relationships between them (Vignal, Mathevon, \&

83 Mottin, 2004).

84 The zebra finch (Taeniopygia guttata) is a social species native to Australia and is monogamous.

85 This songbird forms lifelong pair-bonds (Zann, 1996), and partners are inseparable even outside of 86 the breeding season. In the wild, zebra finches are usually found in small groups, and the pair is 87 the primary social unit (McGowan, Mariette, \& Griffith, 2015). This species extensively uses 88 acoustic communication during social interactions: groups gather in 'social' trees near watering 89 points or feeding areas (Zann, 1996) in which they produce a background sound composed of 90 calls and songs. Some studies have started to focus on vocal dynamics in this species (Elie, 91 Soula, Mathevon, \& Vignal, 2011; Fernandez, Mariette, Vignal, \& Soula, 2016; Gill, Goymann, Ter 92 Maat, \& Gahr, 2015; Perez, Fernandez, Griffith, Vignal, \& Soula, 2015; Villain, Fernandez, 93 Bouchut, Soula, \& Vignal, 2016). Because of the amount of accumulated knowledge on both 94 behavioral and neurobiological aspects of its acoustic communication, the zebra finch is an 95 interesting model to study communal vocalization and its relation to the social structure of the 96 group.

98 Here we hypothesized that the organization of group vocal activity might reveal some aspects of 99 group structure, such as group size and composition. Using the zebra finch as a study species, we 100 investigated the impact of group size and composition on proxies of the group vocal activity and 101 synchrony. We also tested the influence of pair-bonds and spatial location on finer characteristics 102 of dyads vocal interactions. One common difficulty encountered when studying an acoustic 103 network is to determine the identity of the caller and thus to obtain an individual tagging of 104 vocalizations. Also, when we want to assess the acoustic network it might be relevant to control 
105 the spatial proximity between individuals. To overcome both of these issues we used a set-up that 106 first locked the birds in a fixed spatial network of our choosing and allowed us an individual tagging 107 of all vocalizations. We used an in-house software suite that automatically detects vocalizations 108 from hours of passive recording. Our setup also allows for an automatic removal of non109 vocalizations (wings or cage noise) using classification.

110 We built groups of identical sex-ratio but that varied in group sizes and social structures 111 (percentage of paired / unpaired birds). We recorded these groups' vocal activity during several 112 hours on several days, and analyzed the vocal sequences resulting from these recordings. 


\section{Materials and Methods}

\section{Subjects and Housing Conditions}

116 We used 88 adult zebra finches (Taeniopygia guttata) in this study: 44 males and 44 females. All

117 birds came from our breeding colony. Before the experiment, unpaired males and unpaired 118 females were housed with individuals of the same sex, and female-male pairs were housed 119 separately in cages (all cages dimensions: $40 \times 40 \times 40 \mathrm{~cm}$ ) equipped with perches and a pool for 120 environmental enrichment. All birds were kept under the same environmental conditions: 121 temperature between 24 and $26^{\circ} \mathrm{C}$, light conditions of $14: 10 \mathrm{~h}$ light-dark, water, seeds and 122 cuttlefish bones ad libitum and supplemented with salad once a week. As zebra finches are 123 opportunistic breeders, all conditions were reunited for them to breed (water restriction is needed 124 to be in non-breeding condition (Prior, Heimovics, \& Soma, 2013). However they did not have 125 access to nest material so they were not breeding at the time of the recording.

127 Recording Protocol

129 The experiment took place from March to May 2014 and from January to February 2015. The day 130 before the experiment, each bird was moved from the rearing room to the experimental room 131 (sound attenuating chamber, $2.22 \mathrm{~m}$ height $\times 1.76 \mathrm{~m}$ width $\times 2.28 \mathrm{~m}$ length, Silence Box model B, 132 Tip Top Wood, France) and was placed in a cage $(40 \times 40 \times 25)$. Microphones (Audio Technica 133 AT803), connected to a recorder (zoom R16), were placed on top of each cage, above the head of 134 the bird and facing downwards, which is the best position to minimize the variability in vocalization 135 amplitude due to the orientation of the bird's head, and thus maximize vocalization detection 136 (Brumm \& Zollinger, 2011). On each recording day, we recorded vocal exchanges during a long 137 period (three to six hours between 10 am and $4 \mathrm{pm}$ ). 
141 We recorded 35 groups of different sizes (two, four, or eight individuals) and different social 142 compositions $(0 \%, 50 \%$, or $100 \%$ of paired birds). Table 1 gives the number of recorded groups 143 per group size and composition, as well as the number of repetitions for each group, and the total 144 number of hours of recording for each combination. We organized the cages so that all birds can 145 be in visual contact with each member of the group (Figure 1). We defined the distance between 146 two birds as one if the two birds' cages were neighbour, two if the two birds' cages were separated 147 by one cage, etc. Pair mates were always put in neighbouring cages to reduce stress. In 4-bird 148 groups, two males were always located in diagonal and two females in diagonal. In 8-bird groups, 149 the positions of males and females were defined at random.

\section{$151 \quad$ Vocalization Extractions}

152 Vocalizations were extracted from recordings using in-house softwares. These programs were

153 written in python (www.python.org) by authors H.A.S. and M.S.A.F using open-source libraries.

154 Briefly, vocalization detection consisted of a pipeline of five stages. The first process was a simple 155 threshold-based sound detection based on a high-pass filtered energy envelope (1024 samples 156 FFT; $441 \mathrm{~Hz}$ sampling; cut-off frequency: $500 \mathrm{~Hz}$ ). During the second stage, each event above the

157 threshold was reconstructed in order to maintain an amplitude range of $90 \%$ compared to the 158 maximum amplitude. Thus, each event was either lengthened or shortened to obtain the same 159 amplitude range during the event. This technique allows a good estimate of a vocal event's 160 duration. The third stage simply merged overlapping event segments. Together, the three first 161 stages produced start, end, and duration values for each sound event detected in the recording.

162 The accuracy of these three first steps was tested in (Elie et al., 2011). 
163 The fourth stage removed double vocalizations - vocalizations produced by one bird and recorded 164 by its microphone but also recorded by the microphones of all other birds of the group - by using 165 energy and delay differences: to attribute a vocalization to a bird, our program detects 166 vocalizations for each microphone. Then we apply the following algorithm: for each vocalization A 167 (start=sA, end=eA) we look if other vocalizations were detected at the same period in other 168 microphones. If for a vocalization $B$ (start $=s B$ ) we have $s B>s A$ but $s B<e A$ ( $B$ is starting after $A$ but 169 is overlapping $A$ ) we look if the energy of vocalization $B$ is higher than the energy of $A$. If this is the 170 case we keep both vocalizations. If not, we remove $B$ (because $B$ is likely to be $A$ recorded in 171 another microphone). We do this for all vocalizations by eliminating them and/or associate them 172 with a track (hence a bird). Thus, when a vocalization was produced without overlap with another 173 vocalization, our program has a success rate of $100 \%$ (see corresponding tests in supplementary 174 figure 1, a human listener would make more mistakes because the time delay between two 175 microphones is very low, i.e. few milliseconds). The main problem occurs when a vocalization B 176 overlaps a vocalization $A(s B<e A)$, and when $B$ is weaker on its microphone $B$ than $A$ is on the 177 microphone $A$. In that case our program considers that $B$ is the same vocalization as $A$, and thus 178 the vocalization B is lost. We have quantified this type of error. The overlapping vocalizations 179 represent around $11 \%$ of the total number of vocalizations. We have a $46 \%$ error rate on 180 overlapping vocalizations, i.e. the software makes a mistake when attributing the overlapping 181 vocalizations in $46 \%$ of the cases (percentages computed over 1200 vocalizations over eight 182 randomly chosen groups). Thus the errors due to overlapping vocalizations increases the final 183 error rate by $5 \%$.

184 This step effectively tagged uniquely each sound event to an individual. The fifth and last stage 185 removed cage or wings noises using a machine learning process. We trained a supervised 186 classifier using a data set composed of 4500 random extracted sounds from all of our data. Each 187 sound was classified by one expert (M.S.A.F.) as "vocalization" or "non- vocalization". The 188 classification was performed on the spectrogram of the sounds reduced to 50ms: the idea was to 
reduce the quantity of information in term of time and frequency, and sample this information in such a way that we got the same amount of information for each vocalization (short or long). The spectrogram matrix was first reduced to the frequencies of interest - between $500 \mathrm{~Hz}$ and $6 \mathrm{kHz}$. 192 Then two cases appeared: if the vocalization duration was longer than $50 \mathrm{~ms}$, we extracted $50 \mathrm{~ms}$ 193 in the middle of the sound event. If duration was lower than $50 \mathrm{~ms}$, we padded with zero to obtain 194 a 50ms sound event with the relevant event in the middle. The spectrogram was then flattened to 195 obtain a one dimensional vector. We trained a Random Forest classifier (Breiman, 2001) using 1961500 sounds as a training set. The validation set was composed of the remaining 3000 sounds. 197 This classifier had an overall rate of error below $10 \%$.

199 Our vocalization extraction yielded over $1,730 \mathrm{k}$ vocalizations (840k for females and $890 \mathrm{k}$ for 200 males) over a total of 441 hours of recording. Supplementary Figures 2(a) and 2(b) show the 201 histograms of vocalization durations for all extracted vocalizations for each sex. These distributions 202 are composed of two modes that likely represent the two main types of vocalizations produced by 203 zebra finches in this context (Zann, 1996): distance calls i.e. the longer and louder calls given by 204 zebra finches, consisting of a harmonic series modulated in frequency as well as amplitude, and 205 tet or stack calls i.e. softer and shorter calls than distance calls, around 50ms (Zann, 1996). Song 206 syllables are also represented in this histogram because they could not be removed from the 207 dataset (see above). We estimated several parameters of the distribution using a sum of two 208 Gaussians:

$$
P(d)=a N\left(\mu_{1}, \sigma_{1}^{2}\right)+(1-a) N\left(\mu_{2}, \sigma_{2}^{2}\right)
$$

209 where $N\left(\mu, \sigma^{2}\right)$ is the normal Gaussian distribution with mean $\mu$ and variance $\sigma^{2}$. The fit was made 210 on the duration histogram (time step=10ms on $0-1 \mathrm{~s}$ interval) with the least square method.

211 Note that individual song syllables (for males) are not discriminated in one category and can be of 212 any duration. Female and male short calls (tet or stack) have similar duration (first mode at 52 ms). 
213 Consistent with the literature, distance calls are shorter in males (120 ms) compared to females 214 (149 ms) (Supplementary Table 1).

215 Because we were interested only in the dynamic of the vocal exchanges, we decided to pool all 216 vocalizations types together in the following analyses.

218 Data Analysis

220 We separated the analysis into four parts described below: general vocal activity and 221 vocalization rate autocorrelation (analysis of long-term vocal dynamics), and the dyads cross222 correlation and turn-taking transitions analysis (analysis of short-term vocal dynamics). 223

224 General vocal activity

225 We computed the main vocal activity metrics namely the number of vocalizations per time unit for 226 each individual. We also defined a burst as a period where the total vocal activity (for all individuals 227 in the group) was $10 \%$ higher than the average vocal activity (taken on the whole recording day). 228 To find the bursts we split the time into six minutes bins with an overlap of one minute. Thus, a 229 burst could not last less than $5 \mathrm{~min}$. We then analyzed the number of bursts per hour and the total 230 vocalizations rate in bursts only.

232 Vocalization rate autocorrelation

233 The vocalization rate autocorrelation gives information about the presence of cycles in the group 234 general vocal activity. For example, it could tell if the variation of vocalization rate presents 235 patterns over time, i.e. waves of collective vocalizations. 
236 We split the time into 180s bins (3min) with an overlap of 90 s $(1 \mathrm{~min} 30)$ and counted the total 237 number of vocalizations in each bin. We then computed the autocorrelation $\mathrm{AC}(\mathrm{T})$ of this signal 238 with the following formula:

$239 \operatorname{ac}(\mathrm{T})=\operatorname{mean}[(\mathrm{S}(\mathrm{t})-\operatorname{mean}(\mathrm{S}))(\mathrm{S}(\mathrm{t}+\mathrm{T})-\operatorname{mean}(\mathrm{S}))]$

240 with the normalization step we have: $A C(T)=a c(T) / a c(0) . t$ is the time bin number, $S(t)$ is the total

241 number of vocalizations in the bin $\mathrm{t}$, and $\mathrm{T}$ is the time lag value, between 0 and $80 \mathrm{~min}$.

242 A peak at time $\mathrm{T}$ in the autocorrelation curve means that the vocalization rate activity presents a 243 cycle of duration $\mathrm{T}$.

245 Cross-correlation

246 We assessed the vocal temporal synchrony between two birds by computing the cross-correlation 247 at $\mathrm{T}=500 \mathrm{~ms}$. To do that we split the time into $500 \mathrm{~ms}$ bins, and each bird signal was one if the bird 248 vocalized within this period, and zero if not. We computed the cross-correlation (CC(T)) of the two 249 birds' signals with the following formula:

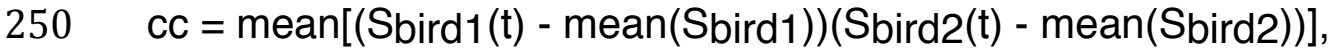

251 with the normalization step we have: $\mathrm{CC}(\mathrm{T})=\mathrm{cc}(\mathrm{t}) /\left(\mathrm{SD}(\mathrm{Sbird} 1)^{\star} \mathrm{SD}(\mathrm{Sbird} 2)\right)$

252 Where Sbird1 and Sbird2 the two birds vocal signals as functions of $t$ (time).

253 For each day of recording we computed cross-correlations for all possible dyads of birds. Two 254 birds having a high cross-correlation value were two birds that were regularly vocalizing closely in 255 time together (within 500ms).

256 We compared cross-correlation between paired/unpaired and neighbour/non-neighbour birds. 
259 In this analysis, we kept only the sequence of callers' identity (without temporal aspect), i.e. the

260 turn-taking. Vocal sequences were obtained with the caller's identity in their order of vocalizing 261 (e.g. ABCA was a sequence of four vocalizations, produced successively by the bird A, then bird 262 B, bird C and finally bird A). We compared the mean transition probabilities for each dyad of birds: 263 between bird $A$ and bird $B$, the mean transition probability is $(\operatorname{prob}(A->B)+\operatorname{prob}(B->A)) / 2$. We 264 compared this measure between paired/unpaired and neighbour/non-neighbour birds.

\section{Statistics}

268 All statistical tests were performed using R software (R Core Team, 2014). Linear mixed models 269 were built with the Imer function ('Ime4' R package (D. Bates, Maechler, Bolker, \& Walker, 2014)) 270 and generalized mixed models were built with the glmer function ('Ime4' R package). Models 271 outputs from Anova ('car' library) and summary functions are presented.

274 Before being interpreted each model was checked, paying particular attention to their residuals.

275 For models with Poisson family, overdispersion was tested, and if the model presented

276 overdispersion we used a negative binomial model. The model validity was also checked with the 277 plotresid function from the 'RVAideMemoire' package before interpreting the model results. When 278 possible, the variance of the data explained by the models was quantified and a conditional 279 coefficient of determination was calculated with 'r.squaredGLMM' function ('MuMln’ R package). 280 
282 We chose to build biologically relevant models and we kept the full model as recommended by 283 Forstmeier \& Schielzeth (2011).

284 Model estimates and confidence intervals

285 When possible we added information about the quantification of the biological effect given by the 286 models. Confidence intervals were computed with the "confint.merMod" function of the Ime4 287 package, with the Wald method.

289 Vocal activity

$290 \quad-\quad$ Number of vocalizations

291 We chose to use the number of vocalizations (NVoc) as the response variable (seen as a count) 292 and we added the recording duration (RecordingDuration) as an offset because all recordings did 293 not last the same time. It was not possible to test the interaction between the group size 294 (GroupSize) and the percentage of pairs in the group (PercentPair) because by definition we did 295 not have a complete crossover design. The other interactions were tested because they were 296 biologically relevant (BirdSex * GroupSize and BirdSex * PercentPair). As a bird could be recorded 297 in several groups, we added the random factor GroupID/BirdID (group identity / bird identity), 298 which took into account the group and the bird in the group. We also had repetitions of recording 299 for each group so we added the repetition number (RepetitionNb) as a random factor:

300 NVoc $\sim$ offset(log(RecordingDuration)) + BirdSex * (GroupSize + PercentPair), 301 random=GroupID/BirdID, RepetitionNb

303 We used a negative binomial model as the model using a Poisson distribution presented over304 dispersion. The interaction between BirdSex and GroupSize was significant, so we separated the 305 dataset into three subsets (for the three group sizes). 
- Number of bursts: We counted the number of bursts and tested the influence of the group size and percentage of pairs on this metric. We built a generalized Poisson model with the recording duration as offset. As for the previous model, it was not possible to test the interaction between the percentage of pairs and the group size because we did not have a complete crossover design. The random factors were the group identity and the repetition number.

313 NumberOfBurst $\sim$ offset(log(RecordingDuration)) + GroupSize + PercentPair, random=GroupID,

314 RepetitionNb

VocRateBurst $\sim$ GroupSize + PercentPair, random=GroupID, RepetitionNb

\section{Vocalizations rate autocorrelation}

We looked at the difference in the time lag of vocalization rate autocorrelation between different group compositions $(0 \%, 50 \%, 100 \%$ of pairs). We detected the secondary maximum peak of each curve from each recording session (the first peak is at 0). For that we used the function

329 'find_peaks_cwt' from the 'signal' python library. We built the following model for each group size: 330 AutocorrelationTimeLag PercentPair random=GroupID, RepetitionNb. 
333 We first build the following general model:

334 CrossCorr $\sim$ GroupSize + PercentPair + Paired, random=GroupID, RepetitionNb, Bird1, Bird2.

335 Explanatory variables had different number of levels depending on group size: the distance 336 between two birds was always 1 for the 2-bird groups, it was either 1 or 2 for the 4-bird groups, 337 and it could be 1, 2, 3, or 4 for the 8-bird groups. Also, the percentage of pairs could only be tested 338 in the 4-bird and 8-bird groups. We thus decided to split the dataset into three sets (one for each 339 group size).

340 We built the following mixed linear models. The variable Paired was "yes" if the bird was paired 341 with another bird in the group, and "no" otherwise. The random factors were the group identity, the 342 repetition number, and the two birds' identities.

344 Group size=2: CrossCorr Paired, random=GroupID, RepetitionNb, Bird1, Bird2.

346 Group size=4: We could not test the interaction between the Paired and Distance variables, 347 because pairs were always at a distance of 1. It was also not possible to test the interaction 348 between Paired and PercentPair because in groups with $100 \%$ of pairs, all birds were paired, and 349 the opposite in groups of $0 \%$ pairs.

350 CrossCorr $\sim$ PercentPair + Paired + Distance + PercentPair:Distance, 351 random=GroupID, RepetitionNb, Bird1, Bird2.

353 Group size=8: The model structure was the same as for the 4-bird groups model above.

354 CrossCorr $\sim$ PercentPair + Paired + Distance + PercentPair:Distance, 355 random=GroupID, RepetitionNb, Bird1, Bird2.

357 We build a second model that included only the data from distance $=1$ :

358 CrossCorr PercentPair * Paired + SameSexDyad + SameSexDyad:Paired, 
random=GroupID, RepetitionNb, Bird1, Bird2

360 The variable SameSexDyad is 'yes' if the corresponding dyad is two females of two males, and 'no'

361 if the corresponding dyad is one male and one female.

364 For the 8-bird groups, the interaction between PercentPair and Distance was significant. We

365 focused on groups with $50 \%$ and $100 \%$ of pairs in order to test the interaction between PercentPair 366 and Paired:

367 CrossCorr $\sim$ PercentPair * Paired + Distance + PercentPair:Distance, 368 random=GroupID, RepetitionNb, Bird1, Bird2

371 Turn-taking transitions probability

372 We compared the mean transition probabilities between paired/unpaired and neighbours/non373 neighbours dyads of birds. As all pairs were set up as neighbours, we had only three possibilities 374 for each dyad of birds in a group with these parameters: UnPaired and NonNeighbour, UnPaired 375 and Neighbour, Paired and Neighbour. As the intrinsic random probability of jumping from a caller i 376 to a caller $\mathrm{j}$ depended on the group size (1/4 for 4-bird groups and 1/8 for 8-bird groups), we 377 studied separately the different group sizes. We had not enough data to compute this metrics on 378 the 2-bird groups. We used the following model for 4-bird and 8-bird groups:

379 MeanTransitionProba $\sim$ PercentPair + PairedNeighb, random=GroupID, RepetitionNb, Bird1, Bird2 380 with PairedNeighb a variable with the three possible levels: UnPaired and NonNeighbour, 381 UnPaired and Neighbour, Paired and Neighbour. 
Individual vocalization rate and burst vocalization rate increase with group size

388 The group size had an effect on the individual vocalization rate: each bird vocalized more when in 389 a larger group. The individual vocalization rate in 8-bird groups was 1.57 times [1.09;2.27] higher 390 than the individual vocalization rate in 2-bird groups. The individual vocalization rate in 4-bird 391 groups was intermediate between the 2-bird and 8-bird groups, but the differences between the 8392 bird vs 4-bird and 4-bird vs 2-bird groups were not significant (Figure 2a, Table 2).

393 The number of bursts was higher in large groups than in small groups. The number of bursts was 3941.27 times [1.03;1.55] higher in 4-bird groups than in 2-bird groups, and 1.47 times [1.22;1.80] 395 higher in 8-bird groups than in 2-bird groups (Figure 2b, Table 3). The overall vocalization rate in 396 bursts was also higher when the group size increased (Figure 2c, Table 3).

Individual vocalization rate decreases when the percentage of pairs increases in the 399 group.

400 In 8-bird groups, we found that the percentage of pairs had an effect on the individual vocalization 401 rate: the individual vocalization rate was lower in groups composed of $100 \%$ of pairs than in 402 groups composed of unpaired birds only. In 8-bird groups, the individual vocalization rate was 1.35 403 times [1.01;1.82] lower in 100\% pairs groups than in 0\% pairs groups (Figure 3a, Table 2).

405 Waves of collective vocalizations have shorter cycles in groups only including 406 unpaired birds 
407 A peak at time $\mathrm{T}$ on the autocorrelation curve means that the vocalization rate activity presents a 408 cycle of duration T. Groups' compositions (0\%, 50\%, 100\% of pairs) were compared at different 409 time lag T (Figure 3b for 8-bird groups, Table 4).

410 In 8-bird groups, the vocalization rate autocorrelation in $0 \%$ pairs groups presented a peak at 411 around $36 \mathrm{~min}+/-13 \mathrm{~min}$ (mean +/- sd). In 50\% pairs groups, the peak was around 46 min $+/-14$ 412 min, and in $100 \%$ pairs groups the peak was around $61 \mathrm{~min}+/-15 \mathrm{~min}$. The time lag tended to be 413 significant between $0 \%$ and $100 \%$ pairs groups (Figure $3 \mathrm{~b}$, Table 4 ), suggesting that $0 \%$ pairs 414 groups had shorter cycles of collective vocalizations than $50 \%$ and $100 \%$ pairs groups.

416 In 4-bird groups, the vocalization rate autocorrelation in $0 \%$ pairs groups presented a peak at 417 around $47 \mathrm{~min}+/-15 \mathrm{~min}$ (mean +/- sd). In 50\% pairs groups, the peak was around 58 min $+/-13$ $418 \mathrm{~min}$, and in $100 \%$ pairs groups the peak was around $60 \mathrm{~min}+/-11 \mathrm{~min}$. We found no significant 419 difference between groups (Figure 3b, Table 4).

\section{Effect of Pair-Bonds and spatial proximity on Short-Term Vocal Dynamics}

Vocal synchrony is higher within pairs and decreases with the distance between individuals

425 We compared cross-correlation between paired/unpaired and neighbour/non-neighbour birds. Two 426 birds showed a high cross-correlation value if they usually vocalized (or stayed silent) closely in 427 time (within 500ms) (i.e "vocal synchrony"). The full model showed no effect of group size or percentage of pairs on dyads' cross-correlation (Table 5). We then split the dataset by group size 429 (see Methods). First, in 2-bird groups, paired and unpaired birds did not differ in cross-correlation 430 (Table 5, Figure 4a). In 4-bird groups, the cross-correlation of a dyad was significantly higher if the 
431 birds were paired, and decreased with the distance (Figure 4b, Table 5). There was no effect of 432 the percentage of pairs in 4-bird groups. Finally, in 8-bird groups, the cross-correlation also 433 decreased with the distance (Figure 4c). At distance $=1$, the cross-correlation between two paired 434 birds was higher than between two unpaired birds (unpaired female/male dyads or same sex 435 unpaired dyads, Supplementary Figure 3, Table 5). The cross-correlation between two paired birds 436 was lower in groups composed of $50 \%$ of pairs than in groups composed of $100 \%$ of pairs (Table

4375 , Figure 4d). The detailed results on the cross-correlation for each group of 8 birds with $100 \%$ of 438 pairs are presented in Supplementary Figure 4.

440 Turn-taking transitions probability is higher within pairs, and is higher between 441 neighbours.

442 We compared the mean transition probabilities between two birds when paired/unpaired and 443 neighbour/non-neighbour. Two birds showed a high mean transition probability if they usually 444 vocalized one after the other. Figure 5 shows the distributions of mean transition probabilities 445 between two birds (paired/unpaired and neighbour/non-neighbour). For both group sizes (4 birds 446 and 8 birds), the mean transition probabilities were higher for UnPaired - Neighbour birds than for 447 UnPaired - NonNeighbour, and even higher for Paired - Neighbour birds. In other words, two 448 paired birds were more likely to vocalize one after the other than two unpaired birds. Within the 449 unpaired birds, two neighbour birds were more likely to vocalize one after the other than two non450 neighbour birds. The statistical results are presented in Table 6. 


\section{Discussion}

453 In this article, we showed that the organization of group vocal activity reveals some aspects of 454 group structure (such as group size and composition) and group acoustic network. On a long-term 455 scale (several minutes to an hour), zebra finches groups synchronize their general vocal activity 456 with waves of collective vocalizations that increase with group size and whose cycles' duration 457 increases with the percentage of pairs in the group. We also showed that the group influences 458 individual vocal activity, with individual vocalization rates increasing with group size and 459 decreasing with the percentage of pairs in the group. On a short-term scale (a few seconds), 460 acoustic interactions are shaped by pair-bonds and distance, as birds preferentially vocalize at the 461 same time (synchrony) or directly after (turn-taking) their partner when it is present, and the 462 nearest neighbour when the partner is not available.

Our setup allowed automatically detecting and extracting vocalizations from hours of passive 465 recording depicting a 'basal' social situation. Our system is able to extract a lot of individual vocalizations (a bit less than $2 \mathrm{M}$ for all experiments combined) with individual tagging. We retrieved a bimodal distribution of duration (well predicted by a sum of two Gaussian) that may relate to the two main types of calls uttered in that context: tets/stacks and distance calls (as well 469 as song syllables).

471 First, on a long-term scale (several minutes to an hour), we saw that large groups have a higher 472 burst rate, and a higher vocal activity within these bursts. This bursting activity can be compared to 473 other waves of collective behaviour (G. Fernandez, Capurro, \& Reboreda, 2003; Pays et al., 474 2007). For example, in Defassa waterbuck, (Kobus ellipsiprymnus defassa), waves of collective vigilance against predators emerge from group members synchronizing scanning and nonscanning bouts and are triggered by allelomimetic effects, i.e. each individual copying its 
neighbour's behaviour (Pays et al., 2007). Focusing on a short-term scale (a few seconds), we saw that neighbouring birds are more likely to vocalize together within a short time window and one after the other. Some individuals could initiate bursts and then neighbours would vocalize as well, triggering waves by an allelomimetic effect. With this hypothesis, the higher bursting activity 481 observed in large groups would be the consequence of the observed increase of the individual 482 vocalization rate. This could be due to a driving effect, because in larger groups there are more 483 individuals to interact with. In killer whales (Orcinus orca), an increase of the production of some 484 vocalization types has been described during socializing and beach-rubbing activities, also 485 corresponding to periods when the group size increases (Ford, 1989). In savanna elephants 486 (Loxodonta africana), it has been shown that the group vocal activity increases when the group 487 size increases (Payne, Thompson, \& Kramer, 2003).

488 One alternative hypothesis to the allelomimetic effect would be that group members exchange 489 information during group vocal interactions. In black-capped chickadees (Poecile atricapillus), 490 dawn choruses can be seen as an interactive communication network. In a recent study, males' 491 dawn chorus singing behaviour was examined by determining the level of song frequency 492 matching between neighbours: male black-capped chickadees were observed having a high level 493 of matching with their neighbours and they also match other individuals simultaneously and 494 sequentially (Foote, Fitzsimmons, Mennill, \& Ratcliffe, 2010).

495 If allelomimetic effects structure group vocal activity, the bursting activity of the group would 496 correlate with the mean individual vocalization rate in the group. To test this prediction, recordings 497 on more groups are needed. On the other hand, if group vocal activity is an information exchange, 498 the group would display strategies to maintain communication efficacy in response to noise. To 499 test this prediction, recordings of groups subjected to background noise playbacks and measures 500 of the response of the group vocal dynamics are needed.

501 As highlighted above, in our study two birds at short distance are more likely to vocalize together 
502 (or stay silent together) within a short time window, but this is even more likely if they are paired. In 503 4-bird groups, neighbour birds were always one male and one female, thus we cannot conclude if 504 the difference of cross-correlation observed is due to the distance or to the intersexual nature of 505 the dyad. Also, we chose to put pairs in neighbour cages to avoid the stress of separation. This is 506 why we could not test whether pairs would show the same behaviour when at longer distance. 507 However the results show that unpaired birds being located at short distance are less likely to 508 vocalize together within a short time window than paired birds at this same distance. In many 509 taxonomic groups, a likely candidate for directing signals to a specific individual is relative signal 510 timing, that is, close temporal association (McGregor, Otter, \& Peake, 2000; Naguib, 1999). In 511 African elephant (Loxodonta africana), it has been shown that females are more likely to produce 512 rumbles shortly after rumbles from other group members. Also, an affiliative relationship with a 513 caller has a strong influence on the probability of a rumble response, whereas the relative 514 dominance rank and reproductive state has no effect (Soltis, Leong, \& Savage, 2005). Another 515 study showed that in Squirrel Monkeys (Saimiri Sciureus), mutually preferred partners exchange 516 chucks more often and with shorter latencies of response than other individuals (Biben, Symmes, 517 \& Masataka, 1986). This result is also consistent with recent studies on zebra finches groups: Ter 518 Maat et al. (2014) show that paired males and females recorded in groups communicate using 519 bilateral stack calling, Gill et al. (2015) show that zebra finches calls occur non-randomly in vocal 520 interactions. Also, Stowell et al. (2016) developed a model that reflects fine details of zebra finches 521 vocal interactions networks, and that especially models the timing and influence strengths between 522 individuals in a group. They showed that within-pair vocal interactions were dominant in the group 523 calling network.

524 The analysis of the transition probabilities between callers also shows that two birds at short 525 distance were more likely to vocalize one after the other. This result on the preferred turn-taking is 526 even stronger if birds are paired. Studies on mammal and bird species with different levels of 527 social coordination show that the social structure of the species relate to different dynamics in their 
528

529

vocal interactions. A recent study shows that in the European starling, the direct and general social context, as well as the individual history, and the internal state of the caller can influence the dynamics of vocalizations in time and structure (Henry, Craig, Lemasson, \& Hausberger, 2015). In our study, we set up the cages in a way that each bird was able to see the whole group, but it was perhaps less easy for a bird to get the visual signals from a bird located far away. If neighbour birds could exchange more visual signals, they might be more likely to answer each other's vocalizations. In human conversation, changes in gazing is one of the signs used to guide conversational turn-taking (Gérard, 1987; Hauser, 1992).

The vocalization rate was lower for the 8-bird groups containing only paired birds than for 8-bird groups containing only unpaired birds. This is consistent with a previous study (Elie et al., 2011) that described zebra finch groups' communal vocalizations (without identification of the callers). Birds involved in a pair-bond could answer preferentially to their partner and less to other members of the group. On the contrary, unpaired birds would have no initial preference and would interact with more birds in the group ("driving effect" suggested above).

Additionally, the autocorrelation analysis showed that the vocal cycling pattern differed with group composition. Groups of eight unpaired birds have shorter cycles that peak around 36min, with a more consistently cycling activity. This is consistent with the previous study from Elie et al. (2011) showing that groups of zebra finches comprising less pairs had shorter vocal cycles.

In larger groups, pairs were more likely to be vocally synchronized in groups containing only pairs than in groups also containing unpaired birds. This could be explained by the fact that, in groups containing unpaired birds, more birds are available for interaction, whereas in groups containing only pairs, each bird may be busy interacting with its own partner as suggested above (Biben et al., 1986; Soltis et al., 2005). 
553 This last result is not observed in intermediate group sizes. One reason could be that 4-bird groups 554 with $50 \%$ of pairs are actually composed of one unique pair, one unpaired male and one unpaired 555 female. The number of unpaired birds potentially available is thus smaller than in 8-bird groups of $55650 \%$ of pairs (composed by two pairs, two unpaired females and two unpaired males).

557 For this same metric, groups of two birds showed no difference between paired or unpaired birds. 558 In these dyads, birds had only one other individual to interact with. Because zebra finches are 559 gregarious birds that use vocal interactions to find and keep social contact (Perez, Fernandez, 560 Griffith, Vignal, \& Soula, 2015; Zann, 1996), they might interact with whoever is available when 561 placed in dyads.

562 In this study we decided to keep all vocalizations types together because among all vocalizations 563 types that zebra finches can produce, in the conditions of our experiment (non-breeding birds and 564 cages at short distances) only three of them were produced: tets, distance calls and songs with a 565 clear bimodal distribution of durations. Separating vocalizations according to duration did not 566 change our results. However it would probably be interesting to study the vocal dynamics by 567 separating the different vocalization types, because the dynamic of vocal exchange could change 568 according to call type, as suggested by (Gill et al., 2015).

569 Also, constraining the birds in one location can be seen as a limitation. Using this protocol we are 570 able to study the acoustic network by constraining the position (in addition to be able to tag 571 individual vocalization more easily). Of course, these position constraints will affect calling 572 dynamics and cannot describe neither situations like group gathering in a social tree, nor 573 interaction contexts like agonistic or physical affiliative behaviours, foraging, etc. So, constraining 574 the spatial position of individuals may have direct and indirect effects on vocal dynamics (Elie et 575 al., 2011). A recent study used devices mounted on the birds to assign vocalizations in bird groups 576 (Gill et al., 2015). This method allows for the study of free-ranging networks of birds in different 577 contexts (agonistic and affiliative behaviours, foraging, and different breeding conditions), and has 
578 the advantage of investigating the calling behaviour of birds behaving freely in a social group.

579 However, it does not give the spatial position of each bird, which can also have an impact on the

580 vocal dynamics. New technologies need to be explored to be able to control for these different

581 aspects at the same time.

582 Here we show that zebra finches groups synchronize their general vocal activity with waves of 583 collective vocalizations, which depend on group size and group composition. Furthermore, at a 584 finer level we show that the group vocal activity is structured by the presence of pairs in the group. 585 Thus we demonstrate that from the acoustic network measured at the individual level we can 586 extract information about the social network. 
Figures legends

591 Figure 1: Schematic of the protocol.

592 Groups of 2, 4, and 8 birds were built, with one bird per cage and one microphone on the top of 593 each cage. Pair mates were put in neighbour cages to reduce stress (distance=1).

Figure 2: Impact of group size on long-term vocal dynamics (a) Effect of group size on individual vocalization rates. (b) Effect of group size on burst rate (number of bursts per hour). (c) 597 Effect of group size on vocalization rate in bursts. Boxes are median, first and third quartiles (Q1 598 and Q3 respectively). The upper whisker is located at the *smaller* of the maximum $\mathrm{x}$ value 599 and Q3 + 1.5 Inter Quartile Range (IQR), whereas the lower whisker is located at the *larger* of 600 the smallest $x$ value and Q1 - 1.5 IQR. Individual points more extreme in value than Q3 + 1.5 IQR 601 are plotted separately at the high end, and those below Q1 - 1.5 IQR are plotted separately on the 602 low end. *: $\mathrm{P}<0.05,{ }^{* *}: \mathrm{P}<0.001,{ }^{* * *}: \mathrm{P}<0.0001$

Figure 3: Impact of the percentage of pairs on the long-term vocal dynamics

(a) Effect of group composition $(0 \%, 50 \%$, and $100 \%$ of pairs in the group) on individual vocalization rates in 8-bird groups. (b) Autocorrelation of the number of vocalizations (time 607 step=3min, overlap=1.5min). Boxes are median, first and third quartiles (Q1 and Q3 respectively). 608 The upper whisker is located at the *smaller* of the maximum $x$ value and Q3 + 1.5 Inter Quartile 609 Range (IQR), whereas the lower whisker is located at the *larger* of the smallest $x$ value and Q1 6101.5 IQR. Individual points more extreme in value than Q3 + 1.5 IQR are plotted separately at the 611 high end, and those below Q1 - 1.5 IQR are plotted separately on the low end.. *: P<0.05 
614 (a) Effect of pair-bonds on cross-correlation in 2-bird groups, (b) Effect of pair-bonds and distance $615(1,2)$ on cross-correlation in 4-bird groups, (c) Effect of pair-bonds and distance $(1,2,3,4)$ on 616 cross-correlation in 8-bird groups, (d) Effect of pair-bonds and group composition (\% of pairs) on 617 cross-correlation in 8-bird groups. Boxes are median, first and third quartiles (Q1 and Q3 618 respectively). The upper whisker is located at the * ${ }^{*}$ maller ${ }^{*}$ of the maximum $x$ value and Q3 +1.5 619 Inter Quartile Range (IQR), whereas the lower whisker is located at the *larger* of the smallest $\mathrm{x}$ 620 value and Q1 - 1.5 IQR. Individual points more extreme in value than Q3 + 1.5 IQR are

621 plotted separately at the high end, and those below Q1 - 1.5 IQR are plotted separately on the 622 low end. *: $\mathrm{P}<0.05,{ }^{* *}: \mathrm{P}<0.001,{ }^{* * *}: \mathrm{P}<0.0001$.

624 Figure 5: Vocal transition probabilities between two birds

Distribution of the average vocal transition probability in (a) 4-bird groups, (b) 8-bird groups. *: $\mathrm{P}<0.05,{ }^{* *}: \mathrm{P}<0.001,{ }^{* * *}: \mathrm{P}<0.0001$

628 Supplementary Figure 1: Detection and attribution of calls to individuals. We tested the 629 detection and attribution of vocalizations to individuals in the three possible positions of a bird in the 630 room (i.e. one angle and two edges, the remaining cages being symmetrical). We put one bird in a 631 cage and recorded it, and repeated the recording in the three possible positions. We had a success 632 rate of $100 \%$ over 240 calls produced (containing tets/stacks and distance calls). The figure 633 provides the sonograms (in green/yellow) and oscillograms (in red) from the 8 microphones for two 634 examples of vocalizations. study. (a) Female vocalizations' durations, (b) Male vocalizations' duration. Tets/stacks and 638 distance calls were detected (resp. first and second peaks) for each sex. Songs were also 639 automatically detected but split so each syllable was considered as a vocalization. 
641 Supplementary Figure 3: Vocal cross-correlation between two birds at distance 1 in 8-bird 642 groups.

643 Effect of sex and pair-bond on cross-correlation at distance 1 in 8-bird groups. Boxes are median, 644 first and third quartiles (Q1 and Q3 respectively). The upper whisker is located at the *smaller* of 645 the maximum $x$ value and Q3 + 1.5 Inter Quartile Range (IQR), whereas the lower whisker is 646 located at the *larger* of the smallest $x$ value and Q1 - 1.5 IQR. Individual points more extreme in 647 value than Q3 + 1.5 IQR are plotted separately at the high end, and those below Q1 - 1.5 IQR are 648 plotted separately on the low end. NS: non-significant, ${ }^{* *}: \mathrm{P}<0.0001$.

Supplementary Figure 4: Acoustic networks for each group in 8-bird groups with $100 \%$ of

pairs. Nodes are individuals $\left(P_{i}\right.$ states for individual in pair $\left.i\right)$. Edges thickness is an affine function of the average vocal cross-correlation on the four recording days for each dyad.

655 Tables legends

656 Table 1: Number of groups for each social composition

657 For each group size and composition, we give the number of groups recorded (i.e. groups with 658 different birds, $X=$ impossible group social composition). In brackets is the number of recording 659 days for each group. The third element is the total number of hours recorded for each combination. 660

661 Table 2: Statistical results of the impact of group composition on individual vocal activity.

662 The full model is presented. Then each group size is analyzed separately and each model is 663 provided. NVocN stands for the number of vocalizations in groups of $\mathrm{N}$ birds.

$664 R^{2} c$ value, which represents the conditional coefficient of determination of the model, is indicated 665 for each model ('r.squaredGLMM' function of 'MuMIn' R package). The table presents the results 
666 from the summary of the model.

668 Table 3: Statistical results of the impact of group composition on bursts activity

$669 R^{2} c$ value, which represents the conditional coefficient of determination of the model, is indicated 670 for each model ('r.squaredGLMM' function of 'MuMIn' R package). The table presents the results 671 from the summary of the model.

673 Table 4: Statistical results of the impact of group composition on the autocorrelation of the number 674 of vocalizations over time

$675 R^{2} c$ value, which represents the conditional coefficient of determination of the model, is indicated 676 for each model ('r.squaredGLMM' function of 'MuMIn' R package). The table presents the results 677 from the summary of the model.

679 Table 5: Statistical results of the impact of group composition on vocal cross-correlation 680 The full model is presented. Then each group size is analyzed separately and each model is 681 provided. CrossCorrN stands for the cross-correlation values in groups of $\mathrm{N}$ birds.

$682 R^{2} c$ value, which represents the conditional coefficient of determination of the model, is indicated 683 for each model ('r.squaredGLMM' function of 'MuMIn' R package). The table presents the results 684 from the summary of the model.

686 Table 6: Statistical results of the impact of group composition on vocal mean transitions 687 probabilities.

688 Groups with four and eight birds are analyzed separately, and each model is provided.

689 MeanTransitionProbaN stands for the mean transition probability values in groups of $\mathrm{N}$ birds.

$690 R^{2} c$ value, which represents the conditional coefficient of determination of the model, is indicated 691 for each model ('r.squaredGLMM' function of 'MuMIn' R package). The table presents the results 
692 from the summary of the model.

693

694

695 Supplementary Table 1: Results on the estimation of vocalization duration parameters.

696 We used a sum of two Gaussians and the fit was made on the duration histogram (time 697 step=10ms on $0-1 \mathrm{~s}$ interval) with the least square method.

698 
699

\section{References}

Adret-Hausberger, M. (1982). Social influences on the whistled songs of starlings. Behavioral Ecology and Sociobiology, 11(4), 241-246. https://doi.org/10.1007/BF00299300

Agetsuma, N. (1995). Foraging synchrony in a group of Yakushima macaques (Macaca fuscata yakui). Folia Primatol, 64, 167-179.

Aubin, T., \& Jouventin, P. (1998). Cocktail-party effect in king penguin colonies. Proc. R. Soc. Lond. B, $265,1665-1673$.

Bates, D., Maechler, M., Bolker, B., \& Walker, S. (2014). Ime4: Linear mixed-effects models using Eigen and S4. R package version 1.1-7. Retrieved from URL: http://CRAN.R-project.org/package=lme4

Bates, M. E., Cropp, B. F., Gonchar, M., \& Knowles, J. (2010). Spatial location influences vocal interactions in bullfrog choruses. J. Acoust. Soc. Am., 127, 2664-2677.

Beauchamp, G. (1992). Diving behavior in surf scoters and Barrow's goldeneyes. Auk, 109, 819-827.

Bebbington, K., \& Hatchwell, B. J. (2015). Coordinated parental provisioning is related to feeding rate and reproductive success in a songbird. Behavioral Ecology. https://doi.org/10.1093/beheco/arv198

Biben, M., Symmes, D., \& Masataka, N. (1986). Temporal and Structural Analysis of Affiliative Vocal Exchanges in Squirrel Monkeys (Saimiri Sciureus). Behaviour, 98(1), 259-273. https://doi.org/10.1163/156853986X00991

Black, J. M., \& Hulme, M. (1996). Partnerships in Birds: The Study of Monogamy. Oxford University Press, 432 pages.

Blanc, F., \& Thériez, M. (1998). Effects of stocking density on the behaviour and growth of farmed red deer hinds. Appl Anim Behav Sci, 56, 297-307.

Blanc, F., Thériez, M., \& Brelurut, A. (1999). Effects of mixed-species stocking and space allowance on the behaviour and growth of red deer hinds and ewes at pasture. Appl Anim Behav Sci, 63, 4153. 
724 Boucaud, I. C. A., Mariette, M. M., Villain, A. S., \& Vignal, C. (2015). Vocal negotiation over parental care? Partners adjust their time spent incubating based on their acoustic communication at the nest. Biological Journal of the Linnean Society, 117(2), 322-336. https://doi.org/10.1111/bij.12705

Boyd, L., \& Bandi, N. (2002). Reintroduction of takhi, Equus ferus przewal- skii, to Hustai National 728 Park, Mongolia: time budget and synchrony of activity pre- and post-release. Appl Anim Behav Sci, 78, 87-102.

Breiman, L. (2001). Random Forests. Machine Learning, 45(1), 5-32.

Brumm, H., \& Zollinger, S. A. (2011). The evolution of the Lombard effect: 100 years of psychoacoustic research. Behaviour, 148, 1173-1198.

Burt, J. M., \& Vehrencamp, S. L. (2005). Dawn chorus as an interactive communication network. In Animal communication networks (P. K. McGregor, pp. 320-343). Cambridge University Press.

Chivers, D. J. (1974). The siamang in Malaya: a field study of a primate in tropical rain forest. Basel: Karger.

Conradt, L. (1998). Could asynchrony in activity between the sexes cause intersexual social segregation in ruminants? Proc R Soc Lond B, 265, 1359-1363.

Côte, S. D., Schaefer, J. A., \& Messier, F. (1997). Time budgets and synchrony of activities in muskoxen: the influence of sex, age and season. Can J Zool, 75, 1628-1635.

Coulson, J. C. (1966). The influence of the pair-bond and age on the breeding biology of the kittiwake gull Rissa tridactyla. The Journal of Animal Ecology, 35(2), 269-279.

Daan, S., \& Slopsema, S. (1978). Short-term rhythms in foraging behavior of the common vole, Microtus arvalis. J Comp Physiol A, 127, 215-227.

Doutrelant, C., McGregor, P. K., \& Oliveira, R. F. (2001). The effect of an audience on intrasexual communication in male Siamese fighting fish, Betta splendens. Behavioral Ecology, 12(3), 283286. https://doi.org/10.1093/beheco/12.3.283 
750

751

752

753

754

755

756

757

758

759

760

761

762

763

764

765

766

767

768

769

770

771

772

773

774

D’spain, G. L., \& Berger, L. P. (2004). Unusual spatiotemporal patterns in fish chorusing. J. Acoust. Soc. Am., 115, 2559-2559.

Elie, J. E., Mariette, M. M., Soula, H. A., Griffith, S. C., Mathevon, N., \& Vignal, C. (2010). Vocal communication at the nest between mates in wild zebra finches: a private vocal duet? Animal Behaviour, 80(4), 597-605.

Elie, J. E., Soula, H. A., Mathevon, N., \& Vignal, C. (2011). Dynamics of communal vocalizations in a social songbird, the zebra finch (Taeniopygia guttata). The Journal of the Acoustical Society of America, 129(6), 4037-4046.

Evans, C. S., \& Marler, P. (1994). Food calling and audience effects in male chickens, Gallus gallus: Their relationships to food availability, courtship and social facilitation. Animal Behaviour, 47(5), 1159-1170.

Farabaugh, S. M. (1982). The ecological and social significance of duetting. Acoustic Communication in Birds, 2, 85-124.

Fernandez, G., Capurro, A. F., \& Reboreda, J. C. (2003). Effect of group size on individual and collective vigilance in greater rheas. Ethology, 109, 413-425. https://doi.org/10.1046/ j.14390310.2003.00887.x

Fernandez, M. S. A., Mariette, M. M., Vignal, C., \& Soula, H. A. (2016). A New Semi-automated Method for Assessing Avian Acoustic Networks Reveals that Juvenile and Adult Zebra Finches Have Separate Calling Networks. Frontiers in Psychology, 7(1816). https://doi.org/10.3389/fpsyg.2016.01816

Foote, J. R., Fitzsimmons, L. P., Mennill, D. J., \& Ratcliffe, L. M. (2010). Black-capped chickadee dawn choruses are interactive communication networks. Behaviour, 147(10), 1219-1248.

Ford, J. K. B. (1989). Acoustic behaviour of resident killer whales (Orcinus orca) off Vancouver Island, British Columbia. Canadian Journal of Zoology, 67(3), 727-745. https://doi.org/10.1139/z89105 
775 Forslund, P., \& Pärt, T. (1995). Age and reproduction in birds - hypotheses and tests. Trends Ecol Evol, $10,374-378$.

Forstmeier, W., \& Schielzeth, H. (2011). Cryptic multiple hypotheses testing in linear models: overestimated effect sizes and the winner's curse. Behavioral Ecology and Sociobiology, 65(1), 47-55. https://doi.org/10.1007/s00265-010-1038-5

Frommolt, K.-H. (1999). Acoustic structure of chorus howling in wolves and consequences for sound propagation. J. Acoust. Soc. Am, 105, 1203-1203.

Gérard, J. (1987). La conversation et les tours de parole. In Savoir Parler, Savoir Dire, Savoir Communiquer (J. Gérard, pp. 33-37). Neuchâtel-Paris: Delacheaux and Niestlé.

Gerhardt, H. C., \& Klump, G. M. (1988). Masking of acoustic signals by the chorus background noise in the green tree frog: a limitation on mate choice. Animal Behaviour, 36(4), 1247-1249.

Gerkema, M. P., \& Verhulst, S. (1990). Warning against an unseen predator: a functional aspect of synchronous feeding in the common vole, Microtus arvalis. Animal Behaviour, 40, 1169-1178.

Gillingham, M. P., \& Klein, D. R. (1992). Late-winter activity patterns of moose (Alces alces gigas) in Western Alaska. Can J Zool, 70, 293-299.

Gill, L. F., Goymann, W., Ter Maat, A., \& Gahr, M. (2015). Patterns of call communication between group-housed zebra finches change during the breeding cycle. eLife, 4, e07770. https://doi.org/10.7554/eLife.07770

Greenfield, M. D. (1994). Synchronous and alternating choruses in insects and anurans: common mechanisms and diverse functions. Amer. Zool., 34, 605-615.

Hall, M. L. (2004). A review of hypotheses for the functions of avian duetting. Behavioral Ecology and Sociobiology, 55(5), 415-430.

Harrington, F. H., \& Mech, L. D. (1979). Wolf howling and its role in territory maintenance. Behaviour, 68(3), 207-249. 
Hauser, M. D. (1992). A mechanism guiding conversational turn-taking in vervet monkeys and rhesus macaques. In Topics of Primatology, Vol. 1. Human Origins (T. Nishida, F. B. M. de Waal, W. McGrew, P. Marler, and M. Pickford, pp. 235-248). Tokyo: Tokyo University Press.

Hector, A. C. K., Seyfarth, R. M., \& Raleigh, M. J. (1989). Male parental care, female choice and the effect of an audience in vervet monkeys. Animal Behaviour, 38(2), 262-271. https://doi.org/10.1016/S0003-3472(89)80088-0

Henry, L., Craig, A. J., Lemasson, A., \& Hausberger, M. (2015). Social coordination in animal vocal interactions. Is there any evidence of turn-taking? The starling as an animal model. Frontiers in Psychology, 6, 1416. https://doi.org/10.3389/fpsyg.2015.01416

Jones, D. L., Jones, R. L., \& Ratman, R. (2009). Dynamical interactions in a green treefrog chorus. J. Acoust. Soc. Am., 126, 2270-2270.

Kavanagh, M. (1978). The diet and feeding behaviour of Cercopithecus aethiops tantalus. Folia Primatol, 30, 30-63.

Langemann, U., Tavares, J. P., Peake, T. M., \& McGregor, P. K. (2000). Response of Great Tits to Escalating Patterns of Playback. Behaviour, 137(4), 451-471.

Lee, J. W., Kim, H. Y., \& Hatchwell, B. J. (2010). Parental provisioning behaviour in a flock-living passerine, the Vinous-throated Parrotbill Paradoxornis webbianus. Journal of Ornithology, 151, 483-490.

Linnane, M. I., Brereton, A. J., \& Giller, P. S. (2001). Seasonal changes in circadian grazing patterns of Kerry cows (Bos taurus) in semi-feral conditions in Killarney National Park, Co. Kerry, Ireland. Appl Anim Behav Sci, 71, 277-292.

Locascio, J. V. (2004). Diel periodicity of fish sound production in Charlotte Harbor, Florida. J. Acoust. Soc. Am., 116, 2640-2640.

Locascio, J. V., \& Mann, D. A. (2005). Effects of Hurricane Charley on fish chorusing. Biology Letters, 1, 362-365.

Mann, D. A. (2003). Patterns of fish sound production. J. Acoust. Soc. Am., 113, 2275-2275. 
825

826

827

828

829

830

831

832

833

834

835

836

837

838

839

840

841

842

843

844

845

846

847

848

849

Mariette, M. M., \& Griffith, S. C. (2012). Nest visit synchrony is high and correlates with reproductive success in the wild Zebra finch Taeniopygia guttata. Journal of Avian Biology, 43(2), 131-140.

Mariette, M. M., \& Griffith, S. C. (2015). The adaptive significance of provisioning and foraging coordination between breeding partners. The American Naturalist, 185(2), 270-280.

Marshall, V. (2003). The chorus environment and the shape of communication systems in frogs. J. Acoust. Soc. Am., 113, 2275-2275.

Mathevon, N. (1997). Individuality of contact calls in the Greater Flamingo Phoenicopterus ruber and the problem of background noise in a colony. Ibis, 139(3), 513-517. https://doi.org/10.1111/j.1474-919X.1997.tb04667.x

Matos, R. J., \& McGregor, P. K. (2002). The effect of the sex of an audience on maleemale displays of Siamese fighting fish (Betta splendens). Behaviour, 139, 1211e1222.

McComb, K., Packer, C., \& Pusey, A. (1994). Roaring and numerical assessment in contests between groups of female lions, Panthera leo. Animal Behaviour, 47(2), 379-387.

McGowan, L. S. C., Mariette, M. M., \& Griffith, S. C. (2015). The size and composition of social groups in the wild zebra finch. Emu. https://doi.org/http://dx.doi.org/10.1071/MU14059

McGregor, P. K., \& Dabelsteen, T. (1996). Communication networks. In Ecology and evolution of acoustic communication in birds. (Kroodsma D. E., Miller E. H., pp. 409-425). Cornell University Press, Ithaca, N.Y.

McGregor, P. K., \& Horn, A. G. (2014). Communication and social networks. In Animal social networks (Jens Krause, Richard James, Daniel W. Franks, Darren P. Croft). Oxford University Press.

McGregor, P. K., Otter, K. A., \& Peake, T. M. (2000). Communication networks: receiver and signaller perspectives. In Animal signals. Adaptive significance of signalling and signal design in animal communication. (Espmark Y, Amundsen T, Rosenqvist G, pp. 405-416). Trondheim: Tapir.

McMahon, B. F., \& Evans, R. M. (1992). Foraging strategies of American white pelicans. Behaviour, 120(69-89). 
850

851

852

853

854

855

856

857

858

859

860

861

862

863

864

865

866

867

868

869

870

Mennill, D. J., Ratcliffe, L. M., \& Boag, P. T. (2002). Female eavesdropping on male song contests in songbirds. Science, 296(5569), 873.

Mitani, J. C. (1984). Gibbon song duets and intergroup spacing. Behaviour, 92(1-2), 59-96.

Naguib, M. (1999). Effects of song overlapping and alternating on nocturnally singing nightingales. Animal Behaviour, 58, 1061-1067.

Payne, K. B., Thompson, M., \& Kramer, L. (2003). Elephant calling patterns as indicators of group size and composition: the basis for an acoustic monitoring system. African Journal of Ecology, 41(1), 99-107. https://doi.org/10.1046/j.1365-2028.2003.00421.x

Pays, O., Renaud, P.-C., Loisel, P., Petit, M., Gerard, J.-F., \& Jarman, P. (2007). Prey synchronize their vigilant behaviour with other group members. Proc. R. Soc. B, 274, 1287-1291. https://doi.org/doi:10.1098/rspb.2006.0204

Perez, E. C., Fernandez, M. S. A., Griffith, S. C., Vignal, C., \& Soula, H. A. (2015). Impact of visual contact on vocal interaction dynamics of pair-bonded birds. Animal Behaviour, 107, 125-137. https://doi.org/http://dx.doi.org/10.1016/j.anbehav.2015.05.019

Plath, M., Blum, D., Schlupp, I., \& Tiedemann, R. (2008). Audience effect alters mating preferences in a livebearing fish, the Atlantic molly, Poecilia mexicana. Animal Behaviour, 75(1), 21-29. https://doi.org/10.1016/j.anbehav.2007.05.013

Prior, N. H., Heimovics, S. A., \& Soma, K. K. (2013). Effects of water restriction on reproductive physiology and affiliative behavior in an opportunistically breeding and monogamous songbird, the zebra finch. Hormones and Behavior, 63(3), 462-74.

Rasmussen, K. L. R. (1985). Changes in the activity budgets of yellow baboons (Papio cynocephalus) during sexual consortships. Behav Ecol Sociobiol, 17, 161-170.

R Core Team. (2014). R: A Language and Environment for Statistical Computing. Vienna, Austria: R Foundation for Statistical Computing. Retrieved from http://www.R-project.org

Rook, A. J., \& Huckle, C. A. (1995). Synchronization of ingestive behaviour by grazing dairy cows. Anim Sci, 60, 25-30. 
876 Rook, A. J., \& Penning, R. D. (1991). Synchronisation of eating, ruminating and idling activity by grazing sheep. Appl Anim Behav Sci, 32, 157-166.

878 879

880

881

882

883

884

885

886

887

888

889

890

891

892

893

894

895

896

897

898

899

Ruckstuhl, K. E. (1998). Foraging behaviour and sexual segregation in bighorn sheep. Animal Behaviour, 56, 99-106.

Ruckstuhl, K. E. (1999). To synchronise or not to synchronise: a dilemma for young bighorn males? Behaviour, 136, 805-818.

Ruckstuhl, K. E., \& Neuhaus, P. (2001). Behavioral synchrony in ibex groups: effects of age, sex and habitat. Behaviour, 138, 1033-1046.

Saino, N., Fasola, M., \& Waiyaki, E. (1995). Do white pelicans Pelecanus onocrotalus benefit from foraging in flocks using synchronous feeding? Ibis, 137, 227-230.

Schenkeveld, L. E., \& Ydenberg, R. C. (1985). Synchronous diving by surf scoter flocks. Can J Zool, 63, 2516-2519.

Schusterman, R. J. (1978). Vocal communication in pinnipeds. In Behavior of Captive Wild Animals (H. Markowitz and V. J. Stevens (Nelson-Hall, Chicago, IL), pp. 247-307).

Simmons, A. M., Bates, M. E., \& Knowles, J. (2009). Non-random patterns of acoustic interactions in chorusing bullfrogs. J. Acoust. Soc. Am., 126, 2270-2270.

Soltis, J., Leong, K., \& Savage, A. (2005). African elephant vocal communication I: antiphonal calling behaviour among affiliated females. Animal Behaviour, 70(3), 579-587. https://doi.org/doi:10.1016/j.anbehav.2004.11.015

Southall, B. L., Schusterman, R. J., \& Kastak, D. (2003). Acoustic communication ranges for northern elephant seals (Mirounga angustirostris). Aquat. Mamm., 29, 202-213.

Spoon, T. R., Millam, J. R., \& Owings, D. H. (2006). The importance of mate behavioural compatibility in parenting and reproductive success by cockatiels, Nymphicus hollandicus. Animal Behaviour, 71(2), 315-326. 
900 Stowell, D., Gill, L., \& Clayton, D. (2016). Detailed temporal structure of communication networks in groups of songbirds. Journal of The Royal Society Interface, 13(119). https://doi.org/10.1098/rsif.2016.0296

Tayler, J. C. (1953). The grazing behaviour of bullocks under two methods of management. Brit J Anim Behav, 1, 72-77.

Ter Maat, A., Trost, L., Sagunsky, H., Seltmann, S., \& Gahr, M. (2014). Zebra Finch Mates Use Their Forebrain Song System in Unlearned Call Communication. PLoS ONE, 9(10), e109334. https://doi.org/10.1371/journal.pone.0109334

908 Tremblay, Y., \& Cherel, Y. (1999). Synchronous underwater foraging behavior in penguins. Condor, 909 101(179-185).

910 Van Rooij, E., \& Griffith, S. C. (2013). Synchronised provisioning at the nest: parental coordination over care in a socially monogamous species. PeerJ, 1, e232.

912 Vignal, C., Mathevon, N., \& Mottin, S. (2004). Audience drives male songbird response to partner's voice. Nature, 430(6998), 448-451.

914 Villain, A. S., Fernandez, M. S. A., Bouchut, C., Soula, H. A., \& Vignal, C. (2016). Songbird mates change 915 their call structure and intrapair communication at the nest in response to environmental noise. Animal Behaviour, 116, 113-129. https://doi.org/10.1016/j.anbehav.2016.03.009

917 Wilson, R. P., Wilson, M.-P. T., \& McQuaid, L. (1986). Group size in foraging African penguins 918 (Spheniscus demersus). Ethology, 72, 338-341.

919 Zann, R. A. (1996). The Zebra Finch : A Synthesis of Field and Laboratory Studies. OUP Oxford, 335 $920 \quad$ pages. 


\section{Acknowledgments}

This work was supported by an ANR grant (French Agence Nationale de la Recherche, project 'Acoustic Partnership') and an IUF grant (Institut Universitaire de France) to C.V., a joint NSF/ANR e CRCNS grant 'AuComSi' for M.S.A.F. and H.A.S.. We are grateful to Colette Bouchut and Nicolas Boyer for their help at the ENES lab. We also thank Caroline Lothe for her help at Beagle Lab. We thank Ingrid Boucaud for the zebra finch drawing. 
Figure 1

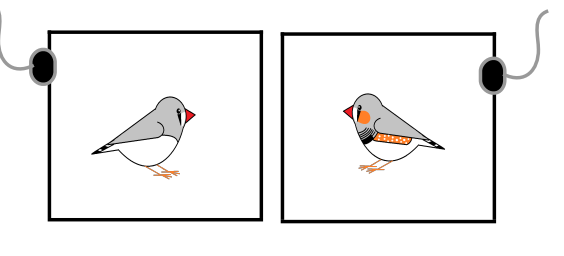

Group size $=2$
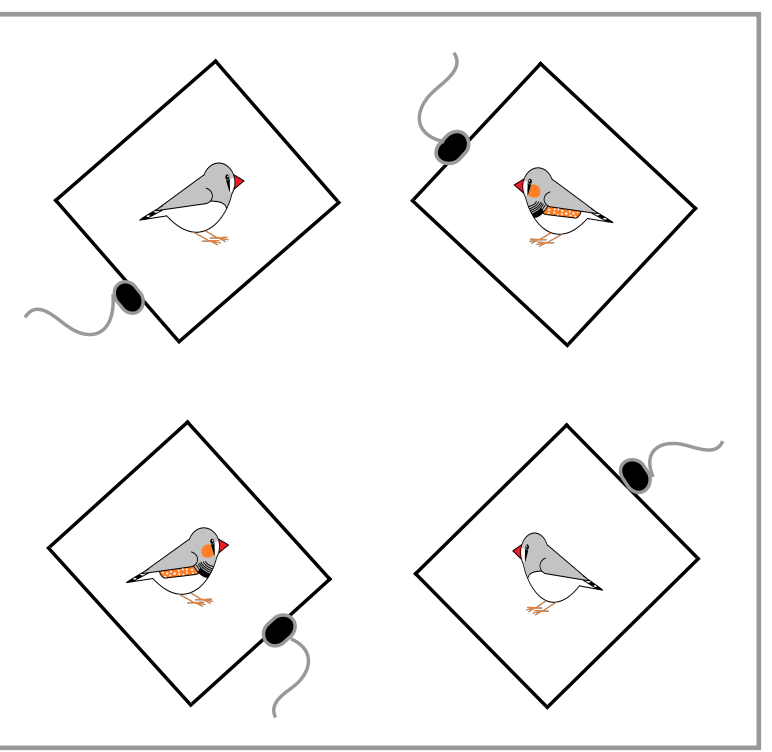

Group size $=4$

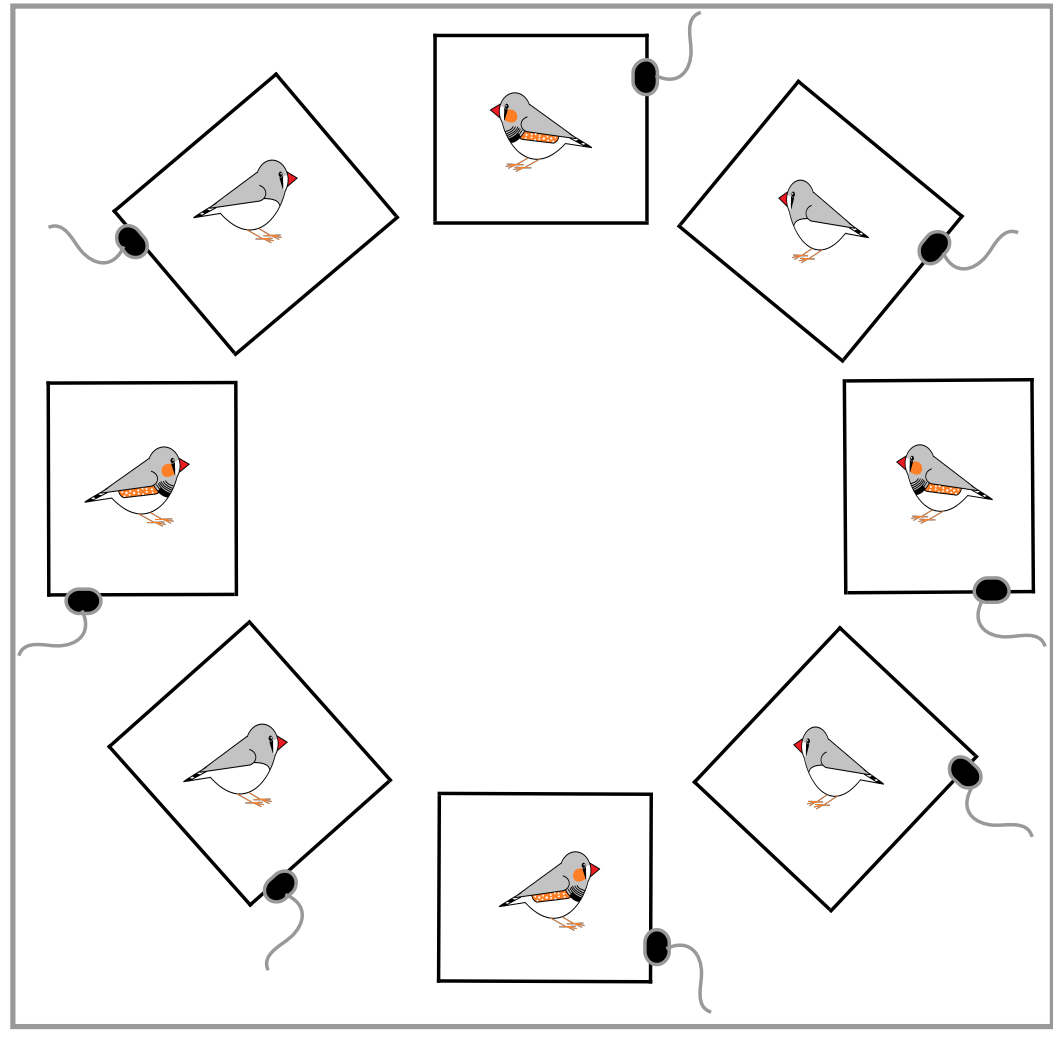

Group size $=8$ 

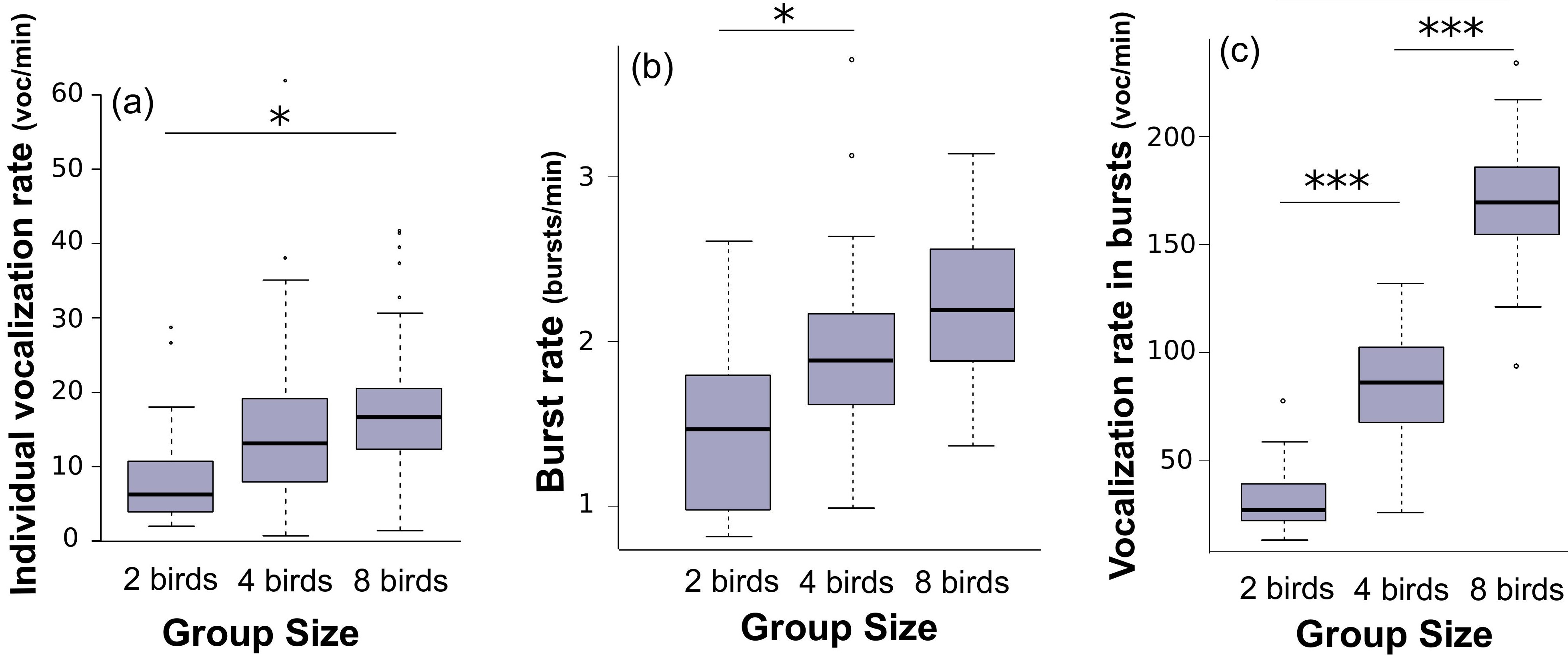
Group Size 

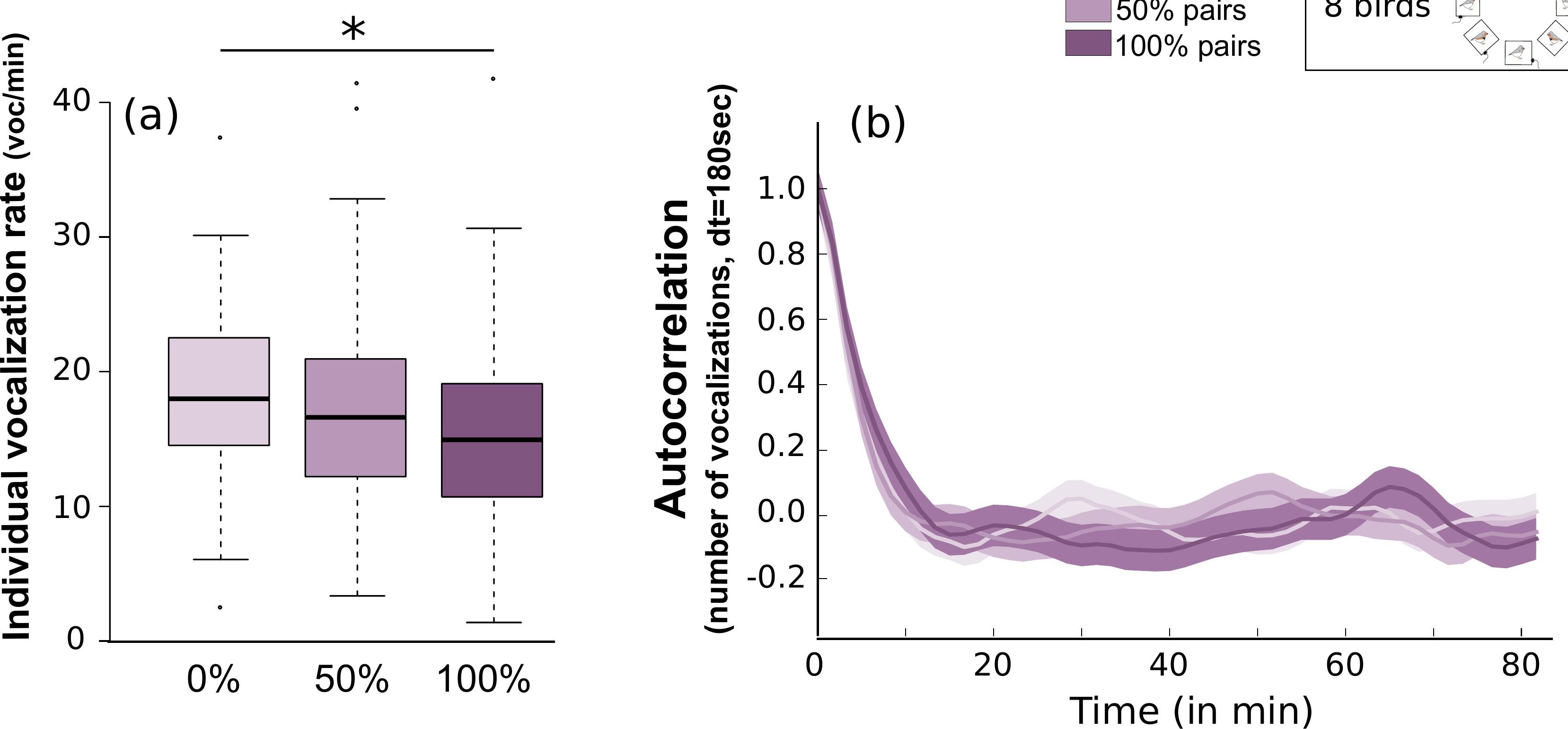


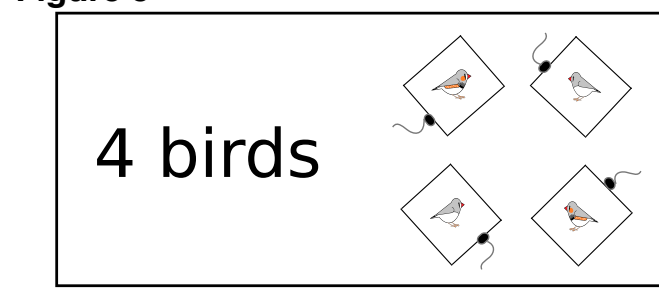

***

4 birds
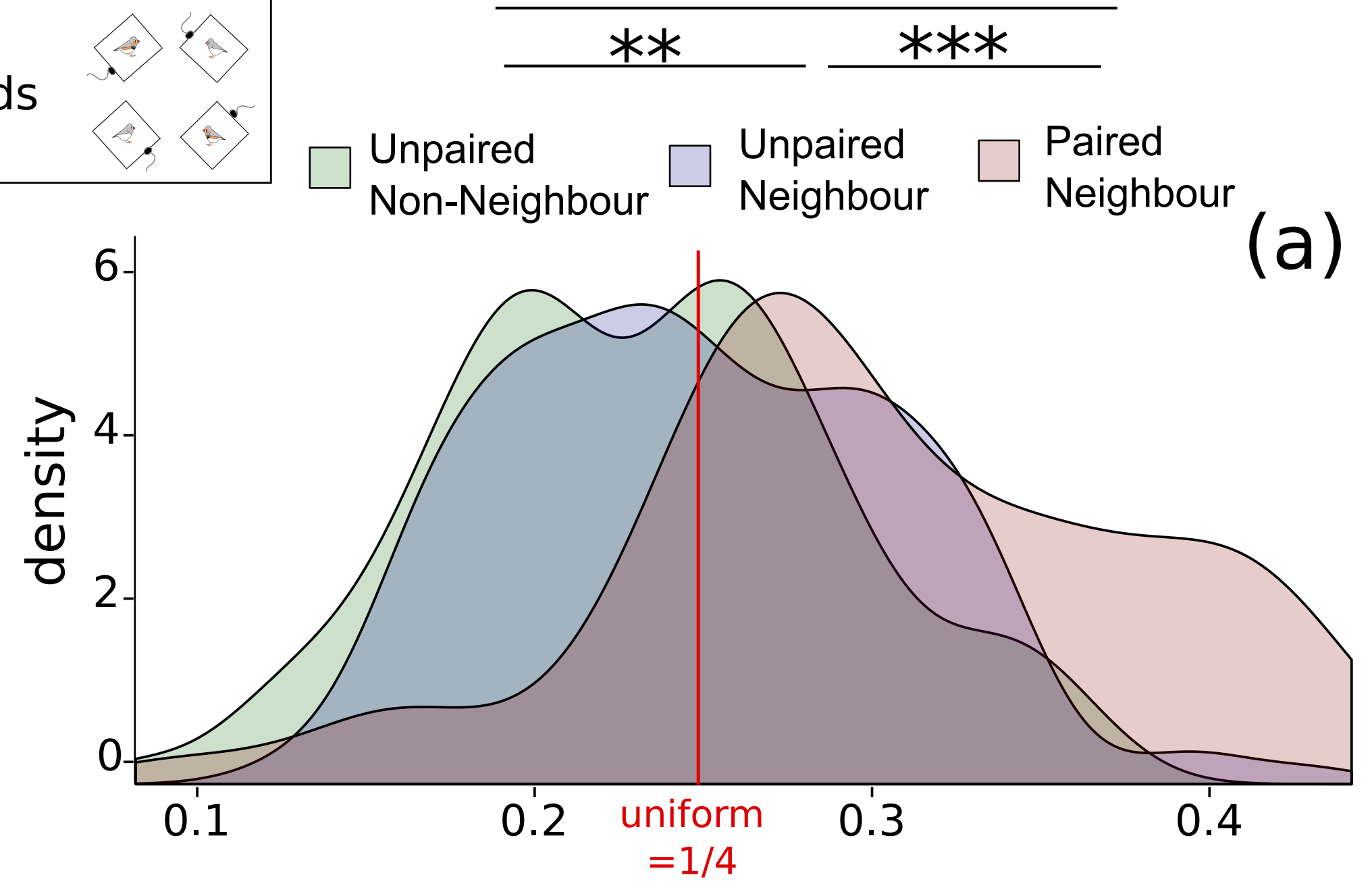

8 birds
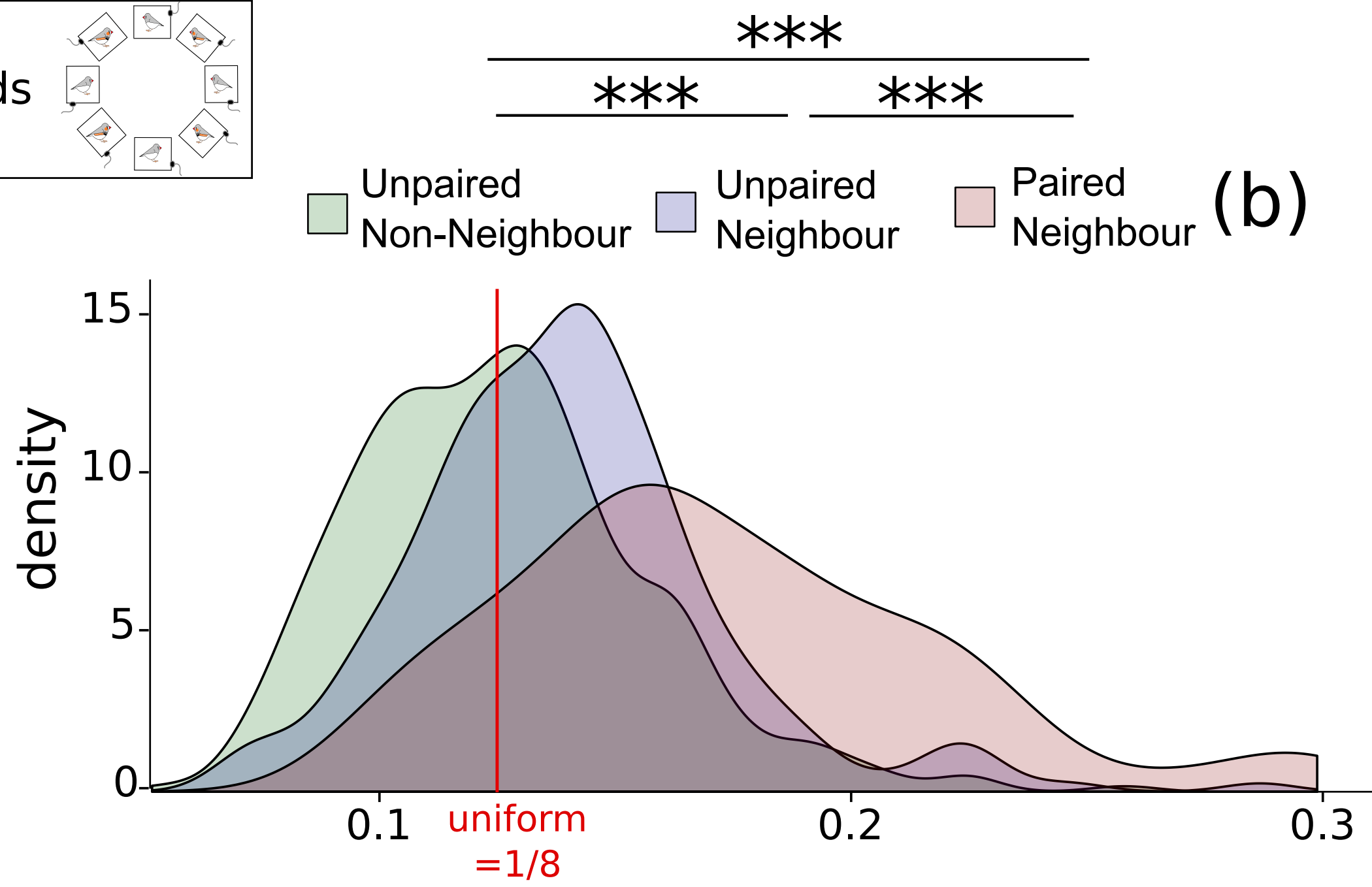

Average of transition probabilities between 2 birds 


\section{Ethical Note}

Experiments were performed under the authorization no. 42-218-0901-38 SV 09 (ENES Laboratory, Direction Départementale des Services Vétérinaires de la Loire) and were in agreement with French and European legislation regarding experiments on animals. 
Table 1

\begin{tabular}{rr|ccc} 
group size & & $0 \%$ paired & $50 \%$ paired & $100 \%$ paired \\
\hline 2 & $6(1)-36 h$ & $X$ & $4(2)+7(1)-89 h$ \\
4 & $3(4)-46 h$ & $3(4)-42 h$ & $3(4)-86 h$ \\
8 & $3(4)-45 h$ & $3(4)-36 h$ & $3(4)-46 h$
\end{tabular}


NVoc $\sim$ offset(log(RecordingDuration)) + BirdSex * (GroupSize + PercentPair ), random=GroupID/BirdID, RepetitionNb

Random effects:

Groups Name

Variance Std.Dev.

GroupID

$0.10291 \quad 0.3208$

BirdID : GroupID

0.06534

0.2556

RepetitionNB

0.0263

0.1622

Residual

0.9343

0.9666

Fixed effects:

\begin{tabular}{lrrrr} 
& Estimate & Std. Error & tvalue & pvalue \\
\hline (Intercept) & 2.3475 & 0.1887 & 12.436 & $<0.0001$ \\
BirdSexM & -0.2145 & 0.1671 & -1.284 & 0.1991 \\
GroupSize4 & 0.1904 & 0.1741 & 1.094 & 0.2742 \\
GroupSize8 & 0.4565 & 0.1856 & 2.46 & 0.0139 \\
PercentPair50 & -0.0538 & 0.2071 & -0.26 & 0.7951 \\
PercentPair100 & -0.2098 & 0.1556 & -1.349 & 0.1774 \\
BirdSexM : GroupSize4 & 0.4405 & 0.1715 & 2.568 & 0.0102 \\
BirdSexM : GroupSize8 & 0.2524 & 0.1656 & 1.524 & 0.1275 \\
BirdSexM : PercentPair50 & -0.0016 & 0.1460 & -0.011 & 0.991 \\
BirdSexM : PercentPair100 & 0.0810 & 0.1243 & 0.652 & 0.5145
\end{tabular}

Pairwise comparisons (Tukey adjustment):

\begin{tabular}{|c|c|c|c|c|}
\hline Contrast & Estimate & SE & zvalue & pvalue \\
\hline GroupSize4 - GroupSize2 & 0.1904 & 0.1741 & 1.094 & 0.517 \\
\hline GroupSize8 - GroupSize2 & 0.4565 & 0.1856 & 2.46 & 0.0368 \\
\hline GroupSize8 - GroupSize4 & 0.2662 & 0.1604 & 1.66 & 0.22 \\
\hline
\end{tabular}

NVoc2 offset(log(RecordingDuration) $)+$ BirdSex * PercentPair, random $=$ GroupID $/$ BirdID

Random effects:

Groups Name

Variance Std.Dev.

GroupID/BirdID

$0.05774 \quad 0.2403$

Residual

$1.10656 \quad 1.0519$

Fixed effects:

(Intercept)

Estimate Std.Error tvalue pvalue

BirdSexM

1.93702

$-0.06731$

0.25458
0.35642

$7.609<0.0001$

PercentPair100

0.29708

0.31197

$-0.189$

0.85

BirdSexM : PercentPair100

$-0.22783$

$0.43006 \quad-0.53$

0.341

0.596 


\begin{tabular}{|c|c|c|c|c|}
\hline \multicolumn{5}{|l|}{$\begin{array}{l}\text { NVoc4 } \sim \text { offset }(10 \\
\text { random=GroupII }\end{array}$} \\
\hline \multicolumn{5}{|l|}{ Random effects: } \\
\hline Groups Name & Variance & Std.Dev. & & \\
\hline GroupID/BirdID & 0.05626 & 0.2372 & & \\
\hline GroupID & 0.16096 & 0.4012 & & \\
\hline RepetitionNB & 0.04658 & 0.2158 & & \\
\hline Residual & 0.92949 & 0.9641 & & \\
\hline \multicolumn{5}{|l|}{ Fixed effects: } \\
\hline & Estimate & Std. Error & tvalue & pvalue \\
\hline$\overline{\text { (Intercept) }}$ & 2.73556 & 0.29264 & 9.348 & $<0.0001$ \\
\hline BirdSexM & 0.07808 & 0.17659 & 0.442 & 0.658 \\
\hline PercentPair50 & -0.30736 & 0.38237 & -0.804 & 0.421 \\
\hline PercentPair100 & -0.41704 & 0.31717 & -1.315 & 0.189 \\
\hline BirdSexM : PercentPair50 & 0.29995 & 0.24969 & 1.201 & 0.23 \\
\hline BirdSexM : PercentPair100 & 0.22832 & 0.21404 & 1.067 & 0.286 \\
\hline
\end{tabular}

\section{NVoc8 offset(log(RecordingDuration) $)+$ BirdSex * PercentPair, randon BirdID, RepetitionNb}

Random effects:

\begin{tabular}{lrr} 
Groups Name & Variance & Std.Dev. \\
\hline BirdID & 0.06748 & 0.2598 \\
GroupID & 0.01339 & 0.1157 \\
RepetitionNB & 0.01741 & 0.1319 \\
Residual & 0.91694 & 0.9576
\end{tabular}

Fixed effects:

Estimate Std. Error tvalue pvalue

\begin{tabular}{lrrrr}
\hline (Intercept) & 2.8465 & 0.1334 & $21.329<0.0001$ \\
BirdSexM & 0.0618 & 0.1281 & 0.483 & 0.6293 \\
PercentPair50 & -0.0706 & 0.1624 & -0.435 & 0.6635 \\
PercentPair100 & -0.2993 & 0.1526 & -1.961 & 0.0499 \\
BirdSexM : PercentPair50 & -0.1042 & 0.1823 & -0.571 & 0.5677 \\
BirdSexM : PercentPair100 & 0.0981 & 0.1718 & 0.571 & 0.5678
\end{tabular}



n=GroupID, 
NumberOfBursts $\sim$ GroupSize + PercentPair, random=GroupID, RepetitionNb

Random effects:

Groups Name Variance Std.Dev.

\begin{tabular}{lrr}
\hline GroupID & 0.00 & 0.00 \\
RepetitionNB & 0.001042 & 0.03228
\end{tabular}

Fixed effects:

\begin{tabular}{lrrrr} 
& Estimate & Std. Error & zvalue & pvalue \\
\hline (Intercept) & -3.6733 & 0.1169 & -31.409 & $<0.0001$ \\
GroupSize4 & 0.2460 & 0.1096 & 2.243 & 0.0248 \\
GroupSize8 & 0.3916 & 0.1232 & 3.177 & 0.0014 \\
PercentPair50 & 0.0215 & 0.1023 & 0.21 & 0.8335 \\
PercentPair100 & -0.0437 & 0.0828 & -0.528 & 0.5971
\end{tabular}

Pairwise comparisons (Tukey adjustment):

\begin{tabular}{lllll} 
Contrast & Estimate & SE & zvalue & pvalue \\
\hline GroupSize4 - GroupSize2 & 0.24605 & 0.10969 & 2.243 & 0.06196 \\
GroupSize8 - GroupSize2 & 0.39161 & 0.12325 & 3.177 & 0.00411 \\
GroupSize8 - GroupSize4 0.14556 & 0.08293 & 1.755 & 0.17993
\end{tabular}

\section{VocalizationRateBurst $\sim$ GroupSize + PercentPair, random $=$ GroupID, RepetitionNb}

$\mathrm{R} 2 \mathrm{c}=0.89$

Random effects:

Groups Name

\begin{tabular}{lll}
\hline GroupID (intercept) & 230.1 & 15.17
\end{tabular}

RepetitionNB (intercept) $\quad 171.3 \quad 13.09$

Residual

$326.6 \quad 18.07$

Fixed effects:

\begin{tabular}{|c|c|c|c|c|c|}
\hline & Estimate & Std. Error & & tvalue & pvalue \\
\hline$\overline{\text { (Intercept) }}$ & 50.083 & 9.111 & 10.37 & 5.497 & $2.31 \mathrm{e}-04$ \\
\hline GroupSize4 & 41.308 & 6.538 & 47.1 & 6.318 & $<0.0001$ \\
\hline GroupSize8 & 121.479 & 7.248 & 40.89 & 16.76 & $5<0.0001$ \\
\hline PercentPair50 & -2.459 & 8.458 & 31 & -0.291 & 0.7731 \\
\hline PercentPair100 & -6.119 & 6.189 & 40.05 & -0.989 & 0.3287 \\
\hline
\end{tabular}

Pairwise comparisons (Tukey adjustment):

Contrast Estimate SE

GroupSize4 - GroupSize2

$\begin{array}{llll}41.308 & 6.538 & 6.318 & <0.0001\end{array}$

GroupSize8 - GroupSize2

121.479

7.248

$16.76<0.0001$

GroupSize8 - GroupSize4

80.171

$6.567 \quad 12.208<0.0001$ 


\section{NbVoc $\sim$ offset(log(RecordingDuration) $)+$ BirdSex * (GroupSize + PercentPair ), random=GroupID/BirdID, RepetitionNb}

Random effects:

\begin{tabular}{lrr} 
Groups Name & Variance & Std.Dev. \\
\hline GroupID & 0.10291 & 0.3208 \\
BirdID : GroupID & 0.06534 & 0.2556 \\
RepetitionNB & 0.0263 & 0.1622 \\
Residual & 0.9343 & 0.9666
\end{tabular}

Fixed effects:

Estimate Std. Error tvalue pvalue

\begin{tabular}{lrrrr}
\hline Intercept) & 2.3475 & 0.1887 & 12.436 & $<0.0001$ \\
BirdSexM & -0.2145 & 0.1671 & -1.284 & 0.1991 \\
GroupSize4 & 0.1904 & 0.1741 & 1.094 & 0.2742 \\
GroupSize8 & 0.4565 & 0.1856 & 2.46 & 0.0139 \\
PercentPair50 & -0.0538 & 0.2071 & -0.26 & 0.7951 \\
PercentPair100 & -0.2098 & 0.1556 & -1.349 & 0.1774 \\
BirdSexM : GroupSize4 & 0.4405 & 0.1715 & 2.568 & 0.0102 \\
BirdSexM : GroupSize8 & 0.2524 & 0.1656 & 1.524 & 0.1275 \\
BirdSexM : PercentPair50 & -0.0016 & 0.1460 & -0.011 & 0.991 \\
BirdSexM : PercentPair100 & 0.0810 & 0.1243 & 0.652 & 0.5145
\end{tabular}

Pairwise comparisons (Tukey adjustment):

\begin{tabular}{|c|c|c|c|c|}
\hline Contrast & Estimate & SE & zvalue & pvalue \\
\hline roupSize4 - Gr & 0.1904 & 0.1741 & 1.094 & 0.517 \\
\hline Size8 - GrounSiz & 0.4565 & 0.1856 & 2.46 & 0.0368 \\
\hline GroupSize8 - GroupSize4 & 0.2662 & 0.1604 & 1.66 & 0.22 \\
\hline
\end{tabular}

NbVoc2 $\sim$ offset(log(RecordingDuration) $)+$ BirdSex * PercentPair,
random=GroupID/BirdID
\begin{tabular}{lcr} 
Random effects: & & \\
Groups Name & Variance & Std.Dev. \\
\hline GroupID/BirdID & 0.05774 & 0.2403 \\
Residual & 1.10656 & 1.0519
\end{tabular}

Fixed effects:

\begin{tabular}{lrrrr} 
& Estimate & Std. Error & tvalue & pvalue \\
\hline (Intercept) & 1.93702 & 0.25458 & 7.609 & $<0.0001$ \\
BirdSexM & -0.06731 & 0.35642 & -0.189 & 0.85 \\
PercentPair100 & 0.29708 & 0.31197 & 0.952 & 0.341 \\
BirdSexM : PercentPair100 & -0.22783 & 0.43006 & -0.53 & 0.596
\end{tabular}




\begin{tabular}{|c|c|c|}
\hline \multicolumn{3}{|l|}{ Random effects: } \\
\hline Groups Name & Variance & Std.Dev. \\
\hline GroupID/BirdID & 0.05626 & 0.2372 \\
\hline GroupID & 0.16096 & 0.4012 \\
\hline RepetitionNB & 0.04658 & 0.2158 \\
\hline Residual & 0.92949 & 0.9641 \\
\hline
\end{tabular}

Fixed effects:

\begin{tabular}{lrrrr} 
& Estimate & Std. Error & tvalue & pvalue \\
\hline (Intercept) & 2.73556 & 0.29264 & $9.348<0.0001$ \\
BirdSexM & 0.07808 & 0.17659 & 0.442 & 0.658 \\
PercentPair50 & -0.30736 & 0.38237 & -0.804 & 0.421 \\
PercentPair100 & -0.41704 & 0.31717 & -1.315 & 0.189 \\
BirdSexM : PercentPair50 & 0.29995 & 0.24969 & 1.201 & 0.23 \\
BirdSexM : PercentPair100 & 0.22832 & 0.21404 & 1.067 & 0.286
\end{tabular}

$\begin{aligned} & \text { NbVoc8 } \sim \text { offset(log(RecordingDuration) })+ \text { BirdSex } \\
& \text { random=GroupID, BirdID, RepetitionNb }\end{aligned}$
\begin{tabular}{lrr} 
Random effects: & & \\
Groups Name & Variance & Std.Dev. \\
\hline BirdID & 0.06748 & 0.2598 \\
GroupID & 0.01339 & 0.1157 \\
RepetitionNB & 0.01741 & 0.1319 \\
Residual & 0.91694 & 0.9576
\end{tabular}

Fixed effects:

\begin{tabular}{lrrrr} 
& Estimate & Std. Error & tvalue & pvalue \\
\hline (Intercept) & 2.8465 & 0.1334 & 21.329 & $<0.0001$ \\
BirdSexM & 0.0618 & 0.1281 & 0.483 & 0.6293 \\
PercentPair50 & -0.0706 & 0.1624 & -0.435 & 0.6635 \\
PercentPair100 & -0.2993 & 0.1526 & -1.961 & 0.0499 \\
BirdSexM : PercentPair50 & -0.1042 & 0.1823 & -0.571 & 0.5677 \\
BirdSexM : PercentPair100 & 0.0981 & 0.1718 & 0.571 & 0.5678
\end{tabular}




8 birds: Autocorrelation $\sim$ PercentPair, random=GroupID $\mathrm{R} 2 \mathrm{c}=0.25$

Random effects:

\begin{tabular}{lll} 
Groups Name & Variance & Std.Dev. \\
\hline GroupID & 24.97 & 4.997 \\
Residual & 184.78 & 13.594
\end{tabular}

Fixed effects:

(Intercept)

Estimate Std. Error

PercentPair50

42.1250

4.8704

0.9739

6.9954

$\begin{array}{ll}5.9960 & 8.649\end{array}$

value pvalue

PercentPair100

13.1045

6.6521

$6.3350 \quad 0.139$

1.32.e-03

PercentPair 100

\section{4 birds: Autocorrelation PercentPair}

$\mathrm{R} 2 \mathrm{c}=0.02$

Fixed effects:

\begin{tabular}{|c|c|c|c|c|c|}
\hline & Estimate & Std. Error & $\mathrm{df}$ & tvalue & pvalue \\
\hline (Intercept) & 52.125 & 3.888 & 13.408 & 13.408 & $<0.0001$ \\
\hline PercentPair50 & 3.500 & 5.498 & 0.637 & 0.637 & 0.528 \\
\hline PercentPair100 & 5.250 & 4.918 & 1.068 & 1.068 & 0.292 \\
\hline
\end{tabular}







\section{CrossCorr $\sim$ GroupSize + PercentPair + Paired, random=GroupID, RepetitionNb, Bird1, Bird2}

\begin{tabular}{llc} 
R2c $=0.79$ & & \\
Random effects: & & \\
Groups Name & Variance & Std.Dev. \\
\hline GroupID & $1.482 \mathrm{e}-02$ & 0.121738 \\
Bird1ID & $3.734 \mathrm{e}-03$ & 0.061110 \\
Bird2ID & $3.124 \mathrm{e}-03$ & 0.055896 \\
RepetitionNB & $5.266 \mathrm{e}-05$ & 0.007257 \\
Residual & $6.564 \mathrm{e}-03$ & 0.081021
\end{tabular}

Fixed effects:

\begin{tabular}{lrrrrr} 
& Estimate & Std. Error & df & tvalue & pvalue \\
\hline (Intercept) & -1.3488 & 0.0511 & 35.3 & -26.389 & $<0.0001$ \\
GroupSize4 & 0.0201 & 0.0539 & 27 & 0.374 & 0.708 \\
GroupSize8 & -0.0067 & 0.0596 & 24.8 & -0.114 & 0.916 \\
PercentPair50 & -0.0621 & 0.0681 & 19.5 & -0.913 & 0.381 \\
PercentPair100 & -0.0841 & 0.0500 & 24.4 & -1.681 & 0.111 \\
PairedYes & 0.1892 & 0.0101 & 1109 & 18.564 & $<0.0001$
\end{tabular}

\section{CrossCorr2 Paired, random=GroupID, RepetitionNb, Bird1, Bird2}

$\mathrm{R} 2 \mathrm{c}=0.87$

Random effects:

\begin{tabular}{lrr} 
Groups Name & Variance & Std.Dev. \\
\hline GroupID & $1.671 \mathrm{e}-04$ & 0.0129 \\
Bird1ID & 0.0195 & 0.1398 \\
Bird2ID & 0.0113 & 0.1065 \\
RepetitionNB & 0.0000 & 0.0000 \\
Residual & 0.0047 & 0.0686
\end{tabular}

Fixed effects:

\begin{tabular}{lcrrrr} 
& Estimate & Std. Error & df & tvalue & pvalue \\
\hline (Intercept) & -1.2680 & 0.0669 & 11.963 & -18.934 & $<0.0001$ \\
Paired & 0.0014 & 0.0403 & 1.781 & 0.035 & 0.976
\end{tabular}




\section{CrossCorr4 $\sim$ PercentPair + Paired + Distance + PercentPair:Distance, random=GroupID, RepetitionNb, Bird1, Bird2}

$\mathrm{R} 2 \mathrm{c}=0.70$

Random effects:

Groups Name Variance Std.Dev.

GroupID

$0.0035 \quad 0.0593$

Bird1ID

$0.0051 \quad 0.0719$

Bird2ID

$0.0043 \quad 0.0660$

RepetitionNB

$0.0000 \quad 0.0000$

Residual

$0.0075 \quad 0.0871$

Fixed effects:

\begin{tabular}{lrrrrr} 
& Estimate & Std. Error & df & tvalue & pvalue \\
\hline Intercept) & -1.3184 & 0.0487 & 10.46 & -27.075 & $<0.0001$ \\
PercentPair50 & -0.0476 & 0.0696 & 10.91 & -0.684 & 0.5081 \\
PercentPair100 & -0.0496 & 0.0589 & 12.38 & -0.842 & 0.4156 \\
PairedYes & 0.1565 & 0.0244 & 139.36 & 6.398 & $<0.0001$ \\
Dist2 & -0.0653 & 0.0240 & 210.69 & -2.715 & 0.0071 \\
PercentPair50:Dist2 & 0.0246 & 0.0341 & 209.11 & 0.723 & 0.4707 \\
PercentPair100:Dist2 & 0.0512 & 0.0308 & 202.26 & 1.662 & 0.0980
\end{tabular}

Pairwise comparisons Paired * Dist (Tukey adjustment):

Contrast

Estimate SE

PairedNo Dist1 - PairedYes Dist1

$-0.1565$

0.02

0.0400

tratio pvalue

PairedNo Dist1 - PairedNo Dist2

0.1965

$0.0132 \quad 208.30$

$-6.208<0.0001$

PairedYes Dist1 - PairedNo Dist2

$0.0259 \quad 143.43$

3.030

0.0145

PairedYes Dist1 - PairedNo Dist2

$0.1965-100$

\section{CrossCorr8 $\sim$ PercentPair + Paired + Distance + PercentPair:Distance, random=GroupID, RepetitionNb, Bird1, Bird2}

$\mathrm{R} 2 \mathrm{c}=0.74$

Random effects:

\begin{tabular}{lcr} 
Groups Name & Variance & Std.Dev. \\
\hline GroupID & $1.344 \mathrm{e}-03$ & 0.0366 \\
Bird1ID & $2.633 \mathrm{e}-03$ & 0.0513 \\
Bird2ID & $2.182 \mathrm{e}-03$ & 0.0467 \\
RepetitionNB & $4.229 \mathrm{e}-05$ & 0.0065 \\
Residual & $2.774 \mathrm{e}-03$ & 0.0526
\end{tabular}

Fixed effects:

(Intercept)

PercentPair50

$\begin{array}{rrrrr}\text { Estimate } & \text { Std. Error } & \text { df } & \text { tvalue } & \text { pvalue } \\ -1.3003 & 0.0268 & 7.4 & -48.503 & <0.0001 \\ 0.0469 & 0.0378 & 7.4 & 1.242 & 0.2524\end{array}$




$\begin{array}{lrrrrr}\text { PercentPair100 } & 0.0327 & 0.0358 & 7.7 & 0.913 & 0.3885 \\ \text { PairedYes } & 0.1042 & 0.0120 & 839.1 & 8.687 & <0.0001 \\ \text { Dist2 } & 0.0236 & 0.0082 & 930.3 & -2.868 & 0.0042 \\ \text { Dist3 } & -0.0679 & 0.0088 & 961.6 & -7.706 & <0.0001 \\ \text { Dist4 } & -0.0359 & 0.0106 & 949.8 & -3.384 & 0.0007 \\ \text { PercentPair50:Dist2 } & -0.0202 & 0.0121 & 928.9 & -1.671 & 0.0951 \\ \text { PercentPair100:Dist2 } & -0.0144 & 0.0121 & 922.4 & -1.186 & 0.2360 \\ \text { PercentPair50:Dist3 } & 0.0208 & 0.0129 & 960.8 & 1.602 & 0.1095 \\ \text { PercentPair100:Dist3 } & 0.0074 & 0.0131 & 958.1 & 0.566 & 0.5713 \\ \text { PercentPair50:Dist4 } & -0.0143 & 0.0155 & 948.6 & -0.927 & 0.3540 \\ \text { PercentPair100:Dist4 } & -0.0245 & 0.0152 & 942.3 & -1.608 & 0.1081\end{array}$

Pairwise comparisons Dist (Tukey adjustment):

Contrast

Estimate SE

Dist2-Dist1

$\begin{array}{llll}-0.0213 & 0.0077 & -2.767 & 0.0285\end{array}$

Dist3-Dist1

$-0.0615$

0.0082

$-7.453<0.0001$

Dist4-Dist1

$\begin{array}{llll}-0.0324 & 0.0099 & -3.262 & 0.0056\end{array}$

Dist3-Dist2

$\begin{array}{llll}-0.0401 & 0.0074 & -5.374<0.0001\end{array}$

Dist4-Dist2

$-0.0110$

0.0092

$-1.197 \quad 0.6252$

Dist4-Dist3

$\begin{array}{llll}0.0290 & 0.0089 & 3.262 & 0.0059\end{array}$

\section{CrossCorr8_Dist1 PercentPair + Paired + SameSexDyad, random=GroupID, RepetitionNb, Bird1, Bird2}

$\mathrm{R} 2 \mathrm{c}=0.72$

Random effects:

Groups Name

Variance Std.Dev.

\begin{tabular}{lll}
\hline GroupID & $1.114 \mathrm{e}-03$ & 0.0333 \\
Bird1ID & $2.426 \mathrm{e}-03$ & 0.0492 \\
Bird2ID & $1.933 \mathrm{e}-03$ & 0.0439 \\
RepetitionNB & $3.491 \mathrm{e}-05$ & 0.0059 \\
Residual & $2.744 \mathrm{e}-03$ & 0.0523
\end{tabular}

Fixed effects:

\begin{tabular}{lrrrrr} 
& Estimate & Std. Error & df & tvalue & pvalue \\
\hline Intercept) & -1.2708 & 0.0246 & 7.2 & -51.575 & $<0.0001$ \\
PercentPair50 & 0.0339 & 0.0346 & 7 & 0.979 & 0.360 \\
PercentPair100 & 0.0090 & 0.0325 & 7.1 & 0.279 & 0.788 \\
PairedYes & 0.1427 & 0.0097 & 793.2 & 14.628 & $<0.0001$ \\
SameSexDyadYes & 0.0016 & 0.0035 & 882.7 & 0.477 & 0.634
\end{tabular}

Pairwise comparisons Paired*SameSexDyad(Tukey adjustment):

Contrast

Unpaired FM - Unpaired FF/MM

Estimate SE

Unpaired FM - Paired FM

$\begin{array}{lllll}-0.0016 & 0.0035 & 888.17 & -0.477 & 0.9642\end{array}$

Unpaired FF/MM - Paired FM

F: female, M: male $\begin{array}{lllll}-0.1427 & 0.0098 & 803.74 & -14.529 & <0.0001\end{array}$ $\begin{array}{lllll}-0.1410 & 0.0098 & 804.26 & -14.358<0.0001\end{array}$ 


\section{CrossCorr8_50\&100\%Pairs PercentPair + Paired + Distance + PercentPair:Distance, random=GroupID, RepetitionNb, Bird1, Bird2}

$\mathrm{R} 2 \mathrm{c}=0.75$

Random effects:

\begin{tabular}{lrr} 
Groups Name & Variance & Std.Dev. \\
\hline GroupID & 0.0011 & 0.0335 \\
Bird1ID & 0.0015 & 0.0396 \\
Bird2ID & 0.0015 & 0.0393 \\
RepetitionNB & $6.291 \mathrm{e}-05$ & 0.0079 \\
Residual & 0.0020 & 0.0448
\end{tabular}

Fixed effects:

\begin{tabular}{lrrrrr} 
& Estimate & Std. Error & df & tvalue & pvalue \\
\hline Intercept) & -1.1553 & 0.0241 & 5.9 & -47.799 & $<0.0001$ \\
PercentPair100 & -0.0425 & 0.0320 & 5.9 & -1.329 & 0.2328 \\
PairedYes & 0.0342 & 0.017 & 521.3 & 2.013 & 0.0446 \\
Dist2 & -0.0470 & 0.0079 & 610.9 & -5.915 & $<0.0001$ \\
Dist3 & -0.0510 & 0.0085 & 635.1 & -5.941 & $<0.0001$ \\
Dist4 & -0.0522 & 0.0098 & 623.7 & -5.287 & $<0.0001$ \\
PercentPair:PairedYes & 0.0850 & 0.0211 & 543.5 & 4.02 & $<0.0001$ \\
PercentPair100:Dist2 & 0.0260 & 0.0113 & 604.6 & 2.292 & 0.0223 \\
PercentPair100:Dist3 & 0.0131 & 0.0123 & 632.1 & 1.068 & 0.2861 \\
PercentPair100:Dist4 & 0.0121 & 0.0138 & 617.7 & 0.874 & 0.3825
\end{tabular}

Pairwise comparisons Paired * Dist (Tukey adjustment):

Contrast

Estimate SE

\begin{tabular}{ll} 
df & tratio pvalue \\
\hline 840.74 & -8.313
\end{tabular}

Unpaired Dist1 - Paired Dist1

$\begin{array}{llll}-0.0935 & 0.0112 & 840.74 & -8.313\end{array}<0.0001$




\section{MeanTransitionProba4 $\sim$ PercentPair + PairedNeighb, random=GroupID, RepetitionNb, Bird1, Bird2}

\begin{tabular}{lrr} 
R2c $=0.70$ & & \\
Random effects: & & \\
Groups Name & Variance & Std.Dev. \\
\hline GroupID & 0.0002 & 0.0164 \\
Bird1ID & 0.0059 & 0.0769 \\
Bird2ID & 0.0059 & 0.0769 \\
RepetitionNB & 0.0000 & 0.0000 \\
Residual & 0.0062 & 0.0791
\end{tabular}

Fixed effects:

\begin{tabular}{lrrrrr}
\hline Intercept) & -1.0009 & 0.0320 & 34.9 & $-31.193<0.0001$ \\
PercentPair50 & 0.0039 & 0.0460 & 34.6 & 0.085 & 0.9328 \\
PercentPair100 & -0.0116 & 0.0372 & 32.9 & -0.313 & 0.7563 \\
UnPairedNeighb & 0.0243 & 0.0079 & 420.5 & 3.051 & 0.0024 \\
PairedNeighb & 0.1477 & 0.0106 & 429.8 & $13.914<0.0001$
\end{tabular}

Multiple comparisons PairedNeighb (Tukey adjustment):

\begin{tabular}{lllll} 
Contrast & Estimate & StdError & z-value & pvalue \\
\hline UnPairedNeighb - UnPairedNonNeighb & 0.0243 & 0.0079 & 3.051 & 0.0062 \\
PairedNeighb - UnPairedNonNeighb & 0.1477 & 0.0106 & 13.914 & $<0.0001$ \\
PairedNeighb - UnPairedNeighb & 0.1233 & 0.0109 & 11.286 & $<0.0001$
\end{tabular}

\section{MeanTransitionProba8 $\sim$ PercentPair + PairedNeighb, random=GroupID, RepetitionNb, Bird1, Bird2}

\begin{tabular}{lrr} 
R2c $=0.68$ & & \\
Random effects: & & \\
Groups Name & Variance & Std.Dev. \\
\hline GroupID & 0.0000 & 0.0000 \\
Bird1ID & 0.0248153 & 0.15753 \\
Bird2ID & 0.0248153 & 0.15753 \\
RepetitionNB & 0.0001199 & 0.01095 \\
Residual & 0.0279268 & 0.16711
\end{tabular}

Fixed effects:

\begin{tabular}{lrrrrr} 
& Estimate & Std. Error & \multicolumn{2}{c}{ tf } & \multicolumn{2}{c}{ pvalue } & \multicolumn{2}{c}{ pvalue } \\
\hline (Intercept) & -2.154 & 0.0463 & 139.6 & $-46.5<0.0001$ \\
PercentPair50 & -0.0260 & 0.065 & 138.1 & -0.401 & 0.689 \\
PercentPair100 & -0.0631 & 0.0608 & 138.7 & -1.037 & 0.301 \\
UnPairedNeighb & 0.1013 & 0.0093 & 1862 & $10.85<0.0001$
\end{tabular}


Supp Figure 1
Click here to download Supplementary material for on-line publication only: SUPP_FIG1.pdf

Supp Figure1
Click here to download Supplementary material for on-line publication only: SUPP_FIG1.pdf

Supp Figure1
Click here to download Supplementary material for on-line publication only: SUPP_FIG1.pdf

(n)

f

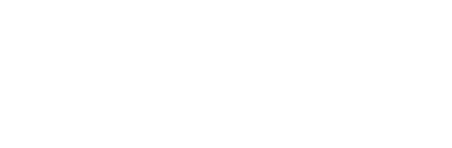
列 (1) (1) (1) (1) (1) (1) (1) (1) (1) (1) (1)

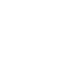


Supp Figure2
Click here to download Supplementary material for on-line publication only: SUPP_FIG2.pdf

Click here to download Supplementary material for on-line publication only: SUPP_FIG2.pdf

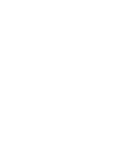

(1)

$\sqrt{20}$

$\sqrt{20}$

-

.

,

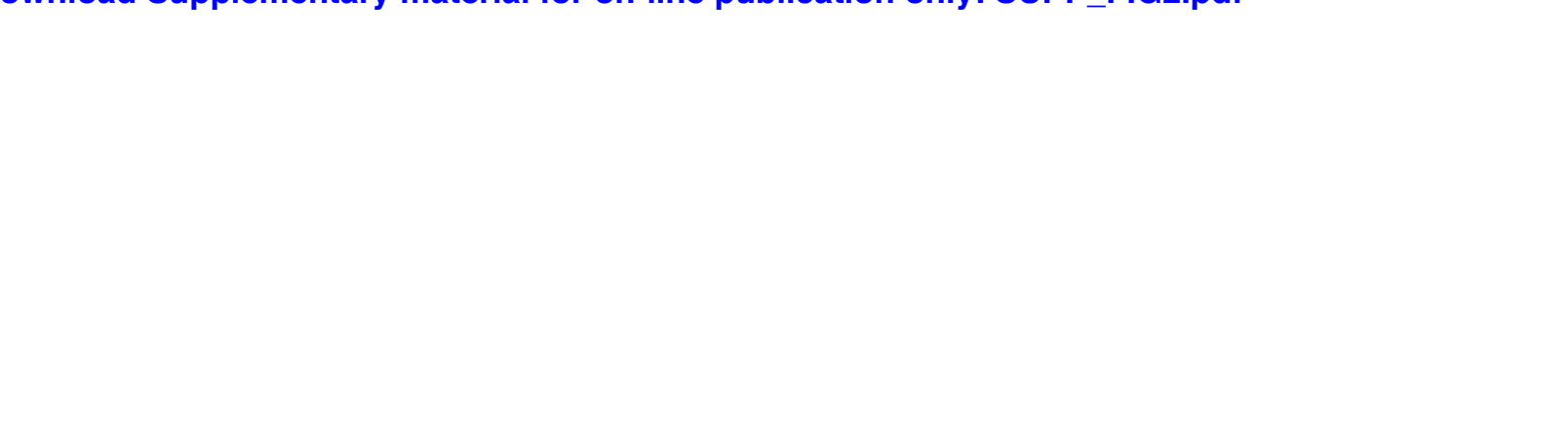

(1)

(1)

(1)

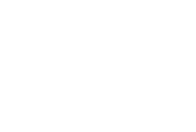

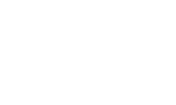




\title{
Supp Figure3
Click here to o \\ Click here to download Supplementary material for on-line publication only: SUPP_FIG3.pdf
} 列

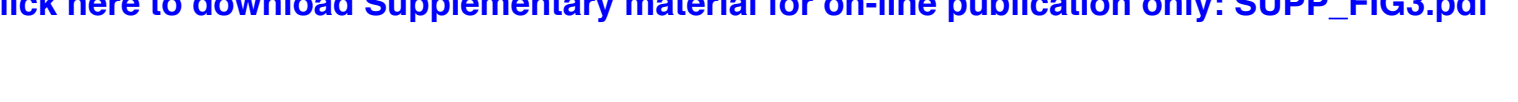

Click here to dow 
Supp Figure 4
Click here to download Supplementary material for on-line publication only: SUPP_FIG4.pdf

for

FIG4.pdf FIG4.pdf

(1)

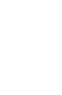
FIG4.pdf (1)

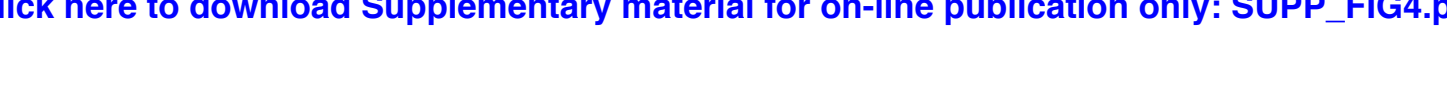
(1) (1) (1) (1) (1) (1) (1) (1) (1) (1) (1) (1) (1) (1) (1) (1) (1) (1) (1) (1) (1) (1) (1) (1) (1) (1) 